\title{
INDIVIDUAL, RELATIONAL, AND CULTURAL CORRELATES \\ OF U.S. LATINO/A COLLEGE STUDENTS' \\ PROSOCIAL BEHAVIORS
}

\author{
A Dissertation \\ presented to \\ the Faculty of the Graduate School \\ at the University of Missouri-Columbia
}

In Partial Fulfillment

of the Requirements for the Degree

Doctor of Philosophy

by

SARAH L. PIEROTTI

Dr. Gustavo Carlo, Dissertation Supervisor

JULY 2020 
The undersigned, appointed by the dean of the Graduate School, have examined the dissertation entitled

\section{INDIVIDUAL, RELATIONAL, AND CULTURAL CORRELATES OF U.S. LATINO/A COLLEGE STUDENTS' PROSOCIAL BEHAVIORS}

presented by Sarah L. Pierotti, a candidate for the degree of doctor of philosophy, and hereby certify that, in their opinion, it is worthy of acceptance.

Professor Gustavo Carlo

Professor Sarah E. Killoren

Professor Jean Ispa

Professor Christi Bergin

Professor Mary B. Eberly Lewis 


\section{DEDICATION}

I would like to dedicate this project to my parents, who have provided support in so many different ways over the years in order for me to pursue any opportunities that I set my sights on, including my higher education. Their love and encouragement have made me the person I am today, and I cannot thank them enough for how they have always been there for me no matter where my ideas take me.

I would also like to dedicate my work to my grandparents-Frank, Lois, Tom, and Ann-for their unwavering encouragement over the years. I especially dedicate this dissertation in memory of my grandfather, Frank Hodas, who passed away earlier this year after a long, fulfilling life and who took so much pride in my education. This work is also dedicated in memory of my godmother, Aunt Lisa, and her work with U.S. Latino/a and Native American families in Wisconsin.

I am very grateful to my friends for being there for me in times of celebration, to lift each other up when needed, and on all of the routine days in between. I feel so fortunate to have you all in my life.

To my coworkers in Human Development and Family Studies at Pennsylvania State University-DuBois, Jess and Marly, and to our students, for reminding me daily through your own work, dedication, and comradery why I chose this field.

To Ariel, for being the most caring, understanding, and supportive partner that I could have ever dreamed of and for already teaching me so much about life. I cannot wait to keep working towards our goals as a team and to continue building a vibrant and gratifying future together. 


\section{ACKNOWLEDGEMENTS}

I would like to acknowledge my doctoral committee for their guidance, not only during the dissertation process, but all throughout graduate school. I would like to recognize my primary advisor, Dr. Gustavo Carlo, for everything he has taught me over the past seven years and for always believing in my potential for growth as a scholar. I have learned so much from him and will carry those lessons with me into the next chapter of my career. I am grateful to Dr. Sarah Killoren for her constant support, mentorship, and understanding. I also owe many thanks to Dr. Jean Ispa for everything that she has taught me, especially about how to be a powerful teacher in the classroom and whose lessons I hope to emulate in my own teaching. I am very appreciative of Dr. Christi Bergin for her constructive feedback and conversations over the years that have always challenged me to become a better scholar. I am also especially grateful to Dr. Mary Eberly Lewis for being a caring mentor who has always believed in me.

Dr. Meredith McGinley deserves special recognition for the mentorship and friendship that she has shared with me during my time in graduate school. I am also grateful to Dr. Deborah Laible for her mentorship and collaboration. I would also like to acknowledge Dr. Celia Brownell, my undergraduate mentor at the University of Pittsburgh, who provided me with a great first research experience and foundation that set me up to be successful in graduate school.

Lastly, I would like to thank my graduate school peers who have shared this journey with me for their teamwork, encouragement, and all of the memories we shared in Gentry Hall. 
TABLE OF CONTENTS

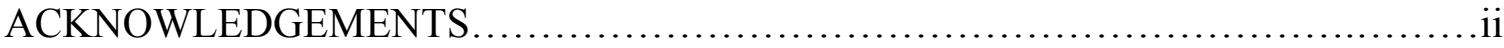

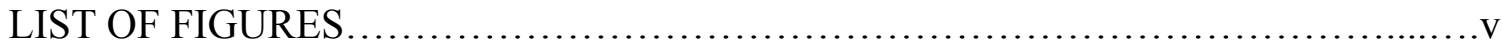

LIST OF TABLES ...........................................................................

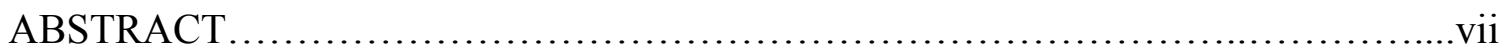

CHAPTER

1. CONCEPTUAL BACKGROUND .........................................

2. LITERATURE REVIEW...............................................4

Existing Integrative Models of Prosocial Behaviors

Cultural Socialization Theories of Prosocial Behaviors

Cultural Socialization Theories of U.S. Latino/a Youth Prosocial Behaviors

Prior Research on Study Variables with U.S. Latino/a Youth

Multidimensionality of Prosocial Behaviors

The Current Study

3. RESEARCH QUESTIONS AND HYPOTHESES........................24

4. RESEARCH DESIGN AND METHODS...................................26

Procedure

Participants

Methods

5. DATA ANALYSIS ............................................................

Preliminary Analyses

Descriptive Analyses 
Variable-Centered Analyses

Person-Centered Analyses

Multigroup Analyses

6. RESULTS

39

Descriptive Statistics and Correlations

Results for Variable-Centered Models

Results for Person-Centered Models

7. DISCUSSION 46

Variable-Centered Findings

Person-Centered Findings

8. OVERALL STRENGTHS, LIMITATIONS, CONCLUSIONS, AND

FUTURE DIRECTIONS

Strengths of the Current Study

Limitations of the Current Study

Conclusions and Future Directions

REFERENCES.

APPENDIX

A. HEURISTIC AND CONCEPTUAL MODELS $\ldots \ldots \ldots \ldots \ldots \ldots \ldots \ldots \ldots . . \ldots . \ldots . \ldots 1$

B. DESCRIPTIVE STATISTICS AND BIVARIATE CORRELATIONS......93

C. VARIABLE-CENTERED MODELS .................................94

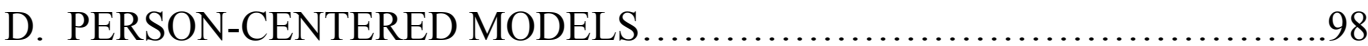

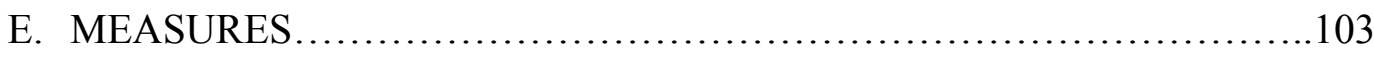

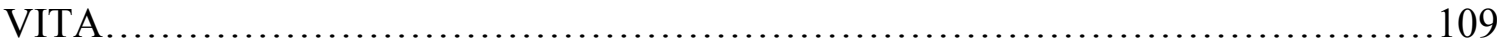




\section{LIST OF FIGURES}

Figure Page

1. Heuristic Model....................................................91

2. Conceptual Model for the Present Study................................92

3. Intervening Mechanisms in the Relations Between Maternal Support and Three Types of Prosocial Behaviors................................. 94

4. Intervening Mechanisms in the Relations Between Paternal Support and Three Types of Prosocial Behaviors.............................. 95

5. Intervening Mechanisms in the Relations Between Maternal Support and Three Unique Targets of Prosocial Behaviors........................96

6. Intervening Mechanisms in the Relations Between Paternal Support and Three Unique Targets of Prosocial Behaviors........................97

7. Latent Profile Indicators............................................... 98 


\section{LIST OF TABLES}

Table $\quad$ Page

1. Correlations and Descriptive Statistics for Study Variables...................93

2. Latent Profile Analysis with Only Maternal Support

Representing Relational Characteristics...............................99

3. Latent Profile Analysis with Only Paternal Support

Representing Relational Characteristics.............................100

4. Latent Profile Analysis with Both Maternal and Paternal Support

Representing Relational Characteristics.............................. 101

5. Differences Across LPA Profiles on LPA Indicators and Correlates..............102 


\begin{abstract}
Prosocial behaviors, or actions intended to benefit others, are important social behaviors that people conduct towards others. These behaviors can be motivated by a host of variables, including individual-level characteristics, relational-level considerations, and culture-specific values. Socioemotive, sociocognitive, and cultural variables have all been studied as important correlates of prosocial behaviors. In addition, maternal and paternal support may play a role in the internalization of these moral motivations. The goal of this study was to test a series of models using both variable-centered and person-centered statistical approaches to investigate how individual-level characteristics, relational-level variables, and culture-specific values both interrelate and simultaneously affect prosocial behaviors. The study used questionnaire measures completed by 250 U.S. Latino/a college students ( $M$ age $=21.0$ years; $62.0 \%$ women $)$. Latent profile analysis and path analysis were used to examine relations among empathic concern, perspective taking, familism, maternal and paternal support, and prosocial behaviors. This research can lend support for culture-specific models of prosocial development that simultaneously account for individual-level, relational-level, and culture-specific characteristics.

Keywords: moral motivations, relational approach, cultural values, prosocial behaviors
\end{abstract}




\section{Chapter 1: Conceptual Background}

Prosocial behaviors (e.g., actions intended to benefit others; Eisenberg et al., 2006) are an important category of social behaviors. Not only do prosocial behaviors provide needed assistance to others, but these behaviors are also linked to positive outcomes for the prosocial actor (Carlo et al., 2014). For college students, engaging in prosocial behaviors may be an especially important indicator of well-being and future success, because this form of behaviors has been found to relate to many other positive outcomes, such as avoiding substance use, academic success, and positive social relationships (Carlo et al., 2014; Carlo et al., 2018).

The number of U.S. Latino/as, which describes people of Latino/a decent living in the United States, is growing, and as of 2018 there are approximately 57.5 million people of Hispanic/Latino/a descent living in the United States (U.S. Census Bureau, 2017). The largest subgroup of U.S. Latino/as is of Mexican descent, and as of 2016, there were approximately 36 million people of Mexican origin living in the United States (Flores, 2017). Despite various positive cultural values being characteristic of U.S. Mexican culture, such as being a moral person, civic responsibility, familism, and respect (Carlo et al., 2009), much prior research with U.S. Latino/a samples has focused on delinquent outcomes and deficit models (García Coll et al., 1996). This has biased the previous literature away from culture-specific understandings of developmental processes in relation to positive outcomes and has led several groups of researchers to call for more research on culture-specific processes on positive development within ethnic-minority families (García Coll et al., 1996; McLoyd et al., 2000; Rafaelli et al., 2005). In order to do so, it is important to remember that to understand behaviors, culture must be 
acknowledged for its primary role in affecting behaviors and development (Markus \& Hamedani, 2007). Moving forward, it is important that researchers continue to emphasize and study positive development in the U.S. Latino/a population and to do so by developing conceptual models that include representations of culture.

According to Bronfenbrenner's bioecological and person-process-context-time models (Bronfenbrenner, 1995, 1999), in order to understand an individual's development, it is important to not only consider individual-level characteristics but also to take into account the relationships that the person has with other people, the context of their environment and culture, and the chronological time period in which they are developing. Therefore, when thinking about how people make decisions about whether to act prosocially or not, there are various motivations and contexts that become relevant. Traditionally, many studies have focused on individual-level characteristics, such as sociocognitive (e.g., perspective taking, prosocial moral reasoning) and socioemotive traits (e.g., empathic concern). In addition, the roles of cultural values (e.g., familism values, respect) and relational variables (e.g., parental support, parental warmth, parental control, parenting practices) have been studied. Some studies and conceptual models have begun to include variables that touch on some or many of these areas (e.g., Carlo et al., 2011; Carlo et al., 2012; Knight et al., 2014; Knight et al., 2016), yet more culturespecific models are needed that theorize on the combined contributions of variables at the individual, relational, and cultural levels. In addition, much of the parenting literature has focused on the role of mothers, but Latino fathers also play an important role in their children's development (Cabrera \& Bradley, 2012). Thus, future theories and conceptual models should make more of an effort to include the role of fathers in prosocial 
development. For this reason, the proposed conceptual models include separate models for mothers and fathers in order to better understand their unique contributions to prosocial development.

Acknowledging that variables at all of these levels are important correlates of prosocial behaviors, the current study aims to provide a more comprehensive theoretical model of U.S. Latino/a young adult prosocial behaviors that includes representations of all of these areas. Prior studies that have looked at correlates at each of these levels have provided important stepping stones to suggest the need to combine all of these spheres of influence into a model that more closely represents the interplay of all of these variables on a daily basis in the real world.

The majority of the existing research on the topic of prosocial behaviors has been conducted using a variable-centered approach, meaning that studies have included various motivations for prosocial behaviors as separate variables and have not looked at how these motivations may work simultaneously to affect a person's prosocial behaviors. The goal of this study was to simultaneously look at individual-level sociocognitive (perspective taking) and socioemotional (empathic concern) characteristics, as well as one representation of U.S. Latino/a culture (familism values) to see how these variables interrelate, as well as how they simultaneously work together to affect prosocial behaviors. In addition, this study includes relational characteristics of the parent-child relationship (maternal and paternal support) to see how individual-level characteristics relate to relational and cultural characteristics to affect prosocial behaviors. 


\section{Chapter 2: Literature Review}

The following section provides an overview of existing literature on prosocial behaviors that is relevant to the current study. It outlines how this study is both in agreement with and differs from previously put forth comprehensive theories of prosocial behaviors to take a more culturally-relative approach.

\section{Existing Integrative Models of Prosocial Behaviors}

\section{Hoffman's Comprehensive Theory of Prosocial Moral Behaviors}

In his updated integrative model of prosocial moral behaviors and development, Hoffman (2001) emphasizes the important role of empathy in driving motivations for moral behaviors. He also highlights the role of cognition, suggesting that both cognition and emotion are important in predicting prosocial behaviors. Hoffman emphasizes that morality can be based on care and concern for others, but his theory also includes justicebased forms of morality. Hoffman's theory is directed towards five specific instances of human behaviors, including being a bystander, being a transgressor, being a virtual transgressor, choosing between multiple moral claimants, and being faced with situations that pit issues of caring and justice against each other. Hoffman states that in order for children's motivations to conduct prosocial behaviors to be strong enough to prompt them to act in these situations that may be conflicting, parents must be active and deliberate in their socialization of the importance of considering and being concerned for other people (Hoffman, 2001). Therefore, it is important to further understand how sociocognitive and socioemotional variables motivate prosocial behaviors and to consider how parents, as well as other socialization agents, teach the importance of these competencies. 
While the heuristic model in the proposed study aligns with Hoffman's views that empathic concern and socialization mechanisms are important for prosocial behaviors, the proposed model differs from Hoffman's comprehensive theory by highlighting the importance of culture. In line with García Coll and colleagues' (1996) call for more culture-specific models of development and Bronfenbrenner's $(1995,1999)$ position that culture plays an important part in development at the macrosystem level, the proposed theory emphasizes the role of cultural values, in conjunction with sociocognitive and socioemotional motives, in prosocial behaviors. Thus, it is important to understand how sociocognitive motives, socioemotional motives, and cultural values are socialized within the specific social group to understand how these motives function.

\section{Socialization Perspectives}

One way in which prosocial behaviors are taught to the next generations is via the process of socialization, and socialization is seen as an important contributor to moral development (Grusec et al., 2014). Socialization refers to the mechanism by which shared values, customs, and ideas are passed from experienced members of the group to others in order to teach them how to become a successful member of the social group (Bugental \& Goodnow, 1998). One area of the socialization literature emphasizes the domains that children learn and are taught within (Grusec et al., 2014). The parent-child relationship, which is the focus of this project, is part of the guided learning domain. In this domain, the parent-child relationship is compared to the relationship between a teacher and student. As such, the teacher (parent) provides the child with opportunities to grow and learn. It is through these learning experiences that children are likely to internalize, or to incorporate into their own ways of thinking, the messages that their parents are trying to 
teach. In turn, children use these lessons as a guide for future behaviors (Grusec et al., 2014).

Part of socialization theory also focuses on the child's point of view in the socialization experience. Grusec and Goodnow (1994) posit that in order for the socialization of a message to occur successfully, it is necessary for the child to accurately interpret the message that the socialization agent (i.e., parent in this case) is trying to convey. Not only is it key that the child accurately interpret the message, but it is also important that that child views the message as reasonable and fitting (Grusec et al., 2014). The child then makes meaning for themselves from these socialization experiences and uses this meaning in the future to guide his or her own behaviors (Grusec et al., 2014). Thus, socialization theory emphasizes the important role that experienced others, such as parents, play in teaching children about values and behaviors.

In Grusec and Goodnow's (1994) model of value internalization, the researchers emphasize that it is more likely that children will internalize the values that their parents are trying to teach if this occurs in a positive parent-child relationship. The authors suggest that parents who are warm, caring, and involved with their children are more likely to have children who internalize their socialization messages (Grusec \& Goodnow, 1994). Thus, qualities of the parent-child relationship are important to include when studying socialization. One way that this can be captured is by measuring children's perceived support from their parents, meaning the existence and expectation of nurturance, affection, companionship, and reassurance of worth within the relationship (Furman \& Buhrmester, 1985).

\section{Social Cognitive Theories}


Another way in which children learn about how to be members of their social group is via social learning. Social cognitive theory, which developed out of social learning theory, reflects how children learn from observing other people (Bandura, 1986). This theory emphasizes that learning, a cognitive process, takes place in social contexts and through interactions with other people. Children learn by observing models as the models engage in behaviors, either by being directly instructed or by learning vicariously. In both cases, children observe the actions of other people around them, learn how to conduct those behaviors, and may replicate those behaviors at a later time (Bandura, 1986). For example, if children have supportive parents, they are likely to learn through their observations and interactions with their parents how to be supportive of others, which would likely encourage future prosocial behaviors.

Empathy-Altruism Hypothesis. The empathy-altruism hypothesis, posited by Batson (1998), applies the principles of social cognitive theory specifically to empathy. In this hypothesis, Batson suggests that when a person who has empathic or sympathetic tendencies observes another person in distress, he or she experiences vicarious physiological arousal by observing the event. Then, this arousal of empathy motivates the person to act in ways that would lessen the other person's suffering. In this selflesslymotivated act, the observer is motivated to act by the desire to help alleviate the other person's suffering (Batson, 1998). In congruence with Hoffman's comprehensive theory (2001), empathy is identified as a key motivator for prosocial action.

\section{Cognitive Developmental Theories}

Other theories on prosocial behaviors take a more strictly cognitive approach. As the study of prosocial behaviors emerged, the field was dominated by cognitive 
developmental theories, such as work by Kohlberg (1976). These theories emphasized the important role of moral cognitions, such as moral reasoning, in driving altruistic behaviors (Kohlberg, 1976). Thus, this position raises the question of how prosocial moral reasoning develops, and perspective taking has been suggested as an important sociocognitive skill that is used in prosocial moral reasoning (Carlo \& Pierotti, 2020). Through cognitive disequilibrium that occurs from being exposed to other people's perspectives, such as role-taking opportunities, these social cognitive traits continue to develop and then affect prosocial behaviors (Carlo \& Pierotti, 2020). Therefore, it is important to understand how cultures vary in these opportunities that they provide children to learn about the perspectives of others and further develop social cognitive traits, which, along with empathic traits, are important in fostering prosocial behaviors.

\section{Cultural Socialization Theories of Prosocial Behaviors}

The process of socialization occurs in each social group, but cultures likely differ in the specific learning opportunities that people have to learn and grow and the culturespecific values that are conveyed during these experiences. Thus, to better understand socialization processes, it is necessary to consider characteristics of the cultural setting in which the socialization occurs.

\section{The Developmental Niche}

Super and Harkness (1986) emphasize the importance of daily interactions and contexts in which children grow up in, and they call this the developmental niche. This idea combines the influence of both culture and relationships with others by highlighting three elements that combine to influence children: the settings and environments that the child grows up within (physical and social), the cultural practices that caregivers use 
when raising the child, and the child's caregivers' own psychology. Thus, the developmental niche framework connects the role of culture with the role of relationships with key socialization agents in shaping the child's experiences growing up. Super and Harkness (1986) indicate that their framework is one way that culture can be accounted for in studying human development.

\section{The Learning Environment}

Super and Harkness are not alone in their emphasis on the importance of the cultural environment that a child is raised in, and in other work this cultural setting has been referred to as the learning environment (Whiting, 1980). In the Whiting (1980) theory of the development of social behaviors across cultures, the learning environment that a child develops within and the characteristics of the people around them are given vital importance. Bringing together elements of the developmental niche and socialization literature, Whiting (1980) suggests that the people with whom a child interacts are responsible for designing and shaping the child's environment, as well as the lessons and behaviors that are taught. Whiting (1980) especially emphasized how this relates to the development of social behaviors. In their Children of Six Cultures Study, the authors found cultural differences in prosocial behaviors based on the type of economy that children were being raised within (Whiting \& Whiting, 1975). In this case, children growing up in subsistence-based economies were found to demonstrate more prosocial behaviors than children growing up in industrialized economies (Whiting \& Whiting, 1975). Yet, it is not the type of economy itself, but rather the different settings, opportunities, and experiences that the economy provides, that likely drive these differences (de Guzman et al., 2008; de Guzman et al., 2005). 


\section{Developmental Affordances}

These differences in opportunities and experiences that children are exposed to have also been called developmental affordances (Calderón-Tena et al., 2011). It is through developmental affordances, meaning opportunities to engage in and practice behaviors, that values and acceptable behaviors continue to be socialized. Furthermore, with more time and more practice through developmental affordances, children adopt these messages as their own and yield the need to be directed by other socialization agents (i.e., parents; Calderón-Tena et al., 2011). Complimentary to the idea of the role of cognitions in influencing prosocial behaviors, during these experiences children may be developing cognitive sets of rules that guide their future behaviors (Knight et al., 2011). It is important to recognize that these cognitive rules are likely based in the values of the culture that the child is growing up in and that socialization agents are choosing to socialize, thus emphasizing potential links and interrelations among culture and cognition.

\section{The Role of Cultural Values}

Culture refers to the system of attitudes, beliefs, values, and behaviors that members of a group share and teach to new members of their group via socialization (Matsumoto, 1996). Thus, one way in which cultures differ from one another is the values that they put forth as the acceptable behaviors of the social group. These cultural values are socialized by experienced others in the culture group, and in turn these values lead to value-related behaviors (Calderón-Tena et al., 2011). One study of 40 countries from around the world found that cultures differ on four major factors: power distance, uncertainty avoidance, individualism, and masculinity (Hofstede, 1984). Research by 
Schwartz (1992) has suggested that there are 56 values that differ by culture, and often subsets of these values are grouped into individualism and collectivism (Schwartz \& Bilsky, 1987). In general, prior research has found that people from collectivist cultures tend to display more prosocial behaviors than people from individualistic cultures (see Knight \& Kagan, 1977), yet the individualism/collectivism distinction does not fully account for the various, nuanced values that differentiate cultures.

It is important to acknowledge that while U.S. Latino/a subgroups likely share many values, each subgroup may endorse values specific to their subgroup. However, the majority of the existing research in the United States has been done with the U.S. Mexican subgroup, and U.S. Mexicans are the largest and fastest growing Latino/a subgroup in the United States (Flores, 2017). The current study will focus on familism values, which have been identified as an important U.S. Mexican cultural value (Knight et al., 2010). To identify cultural values that are perceived by U.S. Mexicans to be important to their culture, Knight and colleagues (2010) conducted focus groups with U.S. Mexican families. Familism emerged from these focus groups as a key cultural value for U.S. Mexican participants. To these participants, familism reflects the desire to maintain close, emotionally-tied family relationships, to provide care for family members, and to refer to the family in making important decisions for the self (Knight et al., 2010). Seeing as the values of a social group are likely to relate to the behaviors that are also encouraged by the group, it is then important to identify the mechanisms by which values affect behaviors.

\section{Cultural Socialization Theories of U.S. Latino/a Youth Prosocial Behaviors}


Although it is expected that cultural values affect prosocial behaviors, the research on how this process occurs is limited (Carlo \& de Guzman, 2009). However, researchers theorize that the socialization of cultural values that emphasize helping and an other-oriented orientation promote the acquisition of sociocognitive traits that are important in predicting helping behaviors (Carlo et al., 2014). Recently, the broader literature on socialization and culture has been integrated into some models of prosocial behaviors specific to U.S. Latino/a youth, which are helpful in guiding the proposed study.

\section{Cultural Value Transmission Model}

In order to explain the process by which cultural values are passed from parents to children, Knight and colleagues (1995) created the cultural value transmission model of Latino/a youth prosocial development. This model emphasizes that culture affects caregivers' own cultural values, which then guide the values that they teach to the next generation. Then, the cultural values that children are taught and internalize affect their own ethnic identity and their likelihood to engage in prosocial behaviors (Knight et al., 1995). Therefore, this model accounts for the socialization mechanism by which the family background and caregivers' culture affects children's prosocial behaviors.

Part of this model has been applied to study familism values with U.S. Mexican early adolescents. The findings support the notion that mothers' familism values relate to her use of familism-based parenting practices, and mothers' familism-based parenting practices are related to adolescents' own perceptions of those practices (Calderón-Tena et al., 2011). Adolescents' perceptions of mothers' familism-based parenting practices are 
related to adolescents' own endorsement of familism values, which are then related to adolescents' prosocial behaviors (Calderón-Tena et al., 2011).

Related longitudinal research by Knight and colleagues (2016) investigated the role of mothers' and fathers' familism values in ethnic socialization practices. In turn, they investigated how these ethnic socialization practices affected children's ethnic identity, familism values, and prosocial behaviors. A number of significant indirect processes emerged from the data that, in general, support the notion that mothers' own familism values when their children are in $5^{\text {th }}$ grade predict their children's prosocial behaviors in $10^{\text {th }}$ grade via ethnic socialization practices when their children are in $5^{\text {th }}$ grade, as well as their children's ethnic identity and familism values measured in $7^{\text {th }}$ grade. For fathers, their familism values when their children were in $5^{\text {th }}$ grade directly predicted their children's familism values in $7^{\text {th }}$ grade, yet there were no significant indirect effects via ethnic socialization or ethnic identity (Knight et al., 2016). Longitudinal studies like these are very promising in their aims to test process models and identify mechanisms by which socialization occurs. More research like Knight and colleagues' (2016) study is needed, especially to continue to delineate the roles of mothers and fathers.

\section{Ecological Stress-Based Models}

Not only is it important to recognize the influence of caregivers' cultural values, but also the broader community context in which the family exists. In Carlo and de Guzman's (2009) ecological stress-based model of Latino/a cultural value transmission, the receiving community and school contexts, life events, and characteristics of the family and child are all included as important antecedents of children's sociocognitive 
and socioemotive development. In this model, sociocognitive and socioemotive variables, such as ethnic identity, moral reasoning, stress appraisals, and empathy, are seen as relating to children's acculturative stress. Acculturative stress is then seen as an intervening mechanism for prosocial behaviors, such that it may foster prosocial behaviors if prosocial behaviors are used as a coping response (Carlo \& de Guzman, 2009).

In Carlo and colleagues' 2014 chapter, a modified version of this model is presented as adapted from Carlo and de Guzman (2009). In this model, antecedent variables (e.g., the receiving community context, family, peer and child characteristics, life events, and the school context) are depicted as predictors of intervening variables at the individual level (e.g., perspective taking, sympathy, ethnic identity, and cultural values). In turn, these individual-level intervening variables are expected to be directly related to prosocial behaviors (Carlo et al., 2014). However, this model does not clearly depict potential bidirectional relations between these variables or account for how all of these contextual, relational, and individual-level characteristics are simultaneously coexisting, interacting, and changing to affect behaviors. This model provides a great conceptual beginning to account for these various levels of influence, and the authors emphasize that incorporating both traditional socialization theories and cultural socialization theories is vital to provide a comprehensive understanding of sociocognitive, socioemotive, and culture-related influences on prosocial behaviors (Carlo et al., 2014). The proposed study aims to build on these ideas to address the simultaneous interrelations of these variables.

\section{Prior Research on Study Variables with U.S. Latino/a Youth}


Although additional research has been done on the associations among the main study variables with samples from other cultural groups, the findings presented here focus only on prior research with U.S. Latino/a participants. The majority of this existing research has been conducted with U.S. Mexican participants, and much more research is needed that addresses potential differences among U.S. Latino/a subgroups. However, the existing research presented below is a starting place to generate new conceptual models and promote more research to address the discussed gaps.

\section{Links between Individual-Level and Cultural-Level Variables}

Carlo and de Guzman (2009) suggest that cultural values and individual-level sociocognitive and socioemotive variables are all important antecedents of prosocial behaviors. Some existing research suggests that perspective taking, empathic concern, and familism values are positively interrelated. Studies have also posited perspective taking to be an important sociocognitive component of empathic concern (Eisenberg, 1991), meaning that in order to feel empathic concern it is also necessary to be able to take the perspective of the other person.

In addition, Feshbach (1987) suggested that people who are both aware and sensitive to the emotions of others (i.e., empathic concern) and who have the sociocognitive traits to understand others' perspectives (i.e., perspective taking), are more likely to engage in prosocial behaviors. In a recent study with U.S. Latino/a adolescents (78\% of the sample identified as U.S. Mexican), perspective taking was directly, positively related to empathic concern (Davis et al., 2018). In a cultural-comparison study that included a subsample of U.S. Mexican early adolescents, researchers found perspective taking to be directly, positively related to sympathy (a variable that combines 
both empathic concern and perspective taking) and to one form of prosocial moral reasoning (hedonistic; Shen et al., 2013). Thus, it is plausible to expect interrelations between sociocognitive and socioemotive variables.

In addition, it is likely that there are relations between cultural values and sociocognitive variables. One recent study was designed to investigate the relations of culture-specific (i.e., familism values) and sociocognitive (i.e., perspective taking, prosocial moral reasoning) variables with a sample of U.S. Mexican early adolescents (Knight et al., 2014). In this study, perspective taking was a significant intervening mechanism between familism values and five types of prosocial behaviors (Knight et al., 2014). This research emphasizes that there are likely complex interrelations between various sociocognitive and socioemotive variables for how they operate as intervening mechanisms to affect prosocial behaviors.

\section{Links between Individual-Level and Relational-Level Variables}

Based on socialization theories presented above, variables that relate to the parent-child relationship are seen as important components of children acquiring sociocognitive and socioemotive traits related to prosocial behaviors. Although no prior studies directly on parental support, empathic concern, and perspective taking with U.S. Latino/a samples were found in a search of the literature, some studies exist on other parenting variables that have been categorized with parental support as positive parenting variables. In a study of U.S. Mexican college students, students were asked to report on their attachment, described as their feelings of whether they have a positive and close relationship, with their closest parental figure (Carlo et al., 2012). Results indicated that closest parent attachment was positively related to empathic concern (Carlo et al., 2012). 
In the cultural-comparison study that included a U.S. Mexican early adolescent subsample described above, maternal induction, meaning the degree of reasoning and explaining that participants reported that their mothers engaged in with them, was also directly, positively related to perspective taking (Shen et al., 2013). In a study with U.S. Mexican early adolescents, maternal induction was found to be positively related to sympathy, which is a composite of empathic concern and perspective taking (Carlo et al., 2011). In addition, sympathy was a significant intervening variable in relations between maternal induction and prosocial behaviors in this study (Carlo et al., 2011). However, the model that was tested was fully saturated, so these findings should be interpreted with caution and replicated in the future. Taken together, this existing body of work indicates that qualities of the parent-child relationship are important in fostering sociocognitive and socioemotive traits, so there are likely significant relations between relational and individual levels of influence.

\section{Links between Relational-Level and Cultural-Level Variables}

It is likely that qualities of the parent-child relationship also affect the acquisition of culture-specific values. Although not conducted with U.S. Latino/as, in a study with families in Mexico, researchers found that parental warmth and support were positively related to children's positive feelings towards their family (Bronstein, 1994). This lends additional evidence to support the idea that positive, warm parenting would foster familism values in families of Mexican descent. In Knight and colleagues' (1995) cultural value transmission model, it was proposed that parents' own cultural values relate to their cultural value-related parenting practices and in turn their children's cultural value endorsement and prosocial behaviors. In a study by Calderón-Tena and 
colleagues (2011), the researchers found support for this model for familism values, suggesting that mothers' own cultural values and the parenting practices that mothers use to promote those specific values influence their adolescents' familism values. In the proposed heuristic model, this relation between qualities of the parent-child relationship and cultural values is depicted by a bidirectional arrow between the relational level and the cultural level.

\section{Links among Individual-Level Variables, Cultural-Level Variables, and Prosocial}

\section{Behaviors}

In addition, it is important to consider how individual-level variables relate to prosocial behaviors. Over time, research evidence has gathered that sociocognitive and socioemotive traits play important roles in driving prosocial behaviors, and a few of these studies have been conducted with U.S. Latino/a samples. In Carlo and colleagues' (2010) study with U.S. Mexican early adolescents, sympathy, which includes empathic concern and perspective taking subscales, was directly and positively related to five forms of prosocial behaviors (emotional, dire, compliant, public, anonymous) and directly and negatively related to altruistic prosocial behaviors. In Carlo and colleagues' (2012) study with U.S. Mexican college students, empathic concern was found to be directly and positively related to five types of prosocial behaviors (dire, emotional, compliant, anonymous, and altruistic).

In a recent study with U.S. Latino/a adolescents (78\% U.S. Mexican), Davis and colleagues (2018) found that perspective taking was directly, positively related to public, emotional, dire, compliant, and anonymous prosocial behaviors, as well as directly, negatively related to altruistic prosocial behaviors. Empathic concern was positively, 
directly related to emotional, dire, altruistic, and compliant prosocial behaviors. There were also significant indirect effects that suggest perspective taking was associated with some types of prosocial behaviors (emotional, dire, altruistic, and compliant) via empathic concern (Davis et al., 2018).

Further, prior research has found cultural values to be related to sociocognitive traits and prosocial behaviors. In a study with U.S. Mexican early adolescents, familism values were directly, positively related to dire, compliant, emotional, and anonymous prosocial behaviors, as well as directly, negatively related to altruistic prosocial behaviors (Knight et al., 2014). In this study, perspective taking was directly, positively related to five forms of prosocial behaviors (dire, compliant, emotional, anonymous, public). In addition, perspective taking acted as a significant intervening mechanism in relations between familism values and prosocial behaviors for five types of prosocial behaviors (dire, compliant, emotional, anonymous, public; Knight et al., 2014). Thus, these findings suggest an interplay between cultural values and sociocognitive traits.

In another study with the same dataset, Calderón-Tena and colleagues (2011) reported U.S. Mexican early adolescents' familism values to be directly, positively related to a composite variable of prosocial behaviors consisting of dire, emotional, and compliant forms of prosocial behaviors. In a longitudinal study with U.S. Mexican youth, Knight and colleagues (2016) found familism values measured in the $7^{\text {th }}$ grade to be directly related to several forms of prosocial behaviors three years later, when participants were in $10^{\text {th }}$ grade. Familism values were directly, positively related to emotional, dire, compliant, anonymous, and public prosocial behaviors, while they were directly, negatively related to altruistic prosocial behaviors (Knight et al., 2016). Thus, 
when taken together, these research findings suggest that sociocognitive and socioemotive traits, as well as endorsement of culture-specific values, are all important correlates of prosocial behaviors for U.S. Latino/a adolescents and could be considered as simultaneous levels of influence.

\section{Links between Relational-Level Variables and Prosocial Behaviors}

In the literature of studies with U.S. Latino/a samples, the majority of research on parenting and prosocial behaviors has been conducted with U.S. Mexican samples. In their study with U.S. Mexican early adolescents, Davis and colleagues (2015) found that for girls, mothers' supportive parenting was directly and positively related to dire, compliant, and altruistic prosocial behaviors, as well as directly and negatively related to public prosocial behaviors. For boys, mothers' supportive parenting was directly, positively related to altruistic prosocial behaviors and directly, negatively related to public prosocial behaviors. In this study, the authors also found a significant indirect effect from mothers' supportive parenting to altruistic prosocial behaviors via the cultural value of traditional gender roles (Davis et al., 2015), thus supporting the idea that characteristics of positive parent-child relationships are related to the internalization of cultural values and prosocial behaviors.

Despite not directly addressing parental support, additional studies on parenting have tapped into some related characteristics of positive parenting (e.g., induction, attachment). In Carlo and colleagues' (2010) study with U.S. Mexican early adolescents, maternal induction was directly, positively related to dire and compliant prosocial behaviors. The role of maternal induction was also present in significant indirect relations to prosocial behaviors via sympathy. The indirect paths were significant and positive for 
maternal induction to dire, compliant, emotional, anonymous and public prosocial behaviors via sympathy, as well as significant and negative for maternal induction to altruistic prosocial behaviors (Carlo et al., 2010).

In Carlo and colleagues' (2012) study with U.S. Mexican college students, closest parent attachment was directly, positively related to empathic concern, which in turn was directly related to anonymous, emotional, dire, compliant, and altruistic prosocial behaviors. However, closest parent attachment was not significantly related to any forms of prosocial behaviors, nor were there significant indirect effects from closest parent attachment to prosocial behaviors via empathic concern. Although closest parent attachment was not directly related to prosocial behaviors, it was related to empathic concern, which was related to prosocial behaviors (Carlo et al., 2012). These findings indicate that parenting variables play a role in prosocial behaviors, both directly and indirectly via fostering sociocognitive and socioemotive traits. More research is needed that may help explain how additional qualities of the parent-child relationship, as well as specific, culture-related parenting practices, affect prosocial behaviors.

\section{Multidimensionality of Prosocial Behaviors}

Prosocial behaviors, meaning actions intended to benefit others (Eisenberg et al., 2006), are important social behaviors that were found in every culture studied in the Children of Six Cultures study (Whiting, 1980). As with any social behaviors, prosocial behaviors are complex and should be recognized as a multidimensional construct rather than a unidimensional construct. For example, the likelihood of a person engaging in prosocial behaviors can vary based on the type of behaviors that the situation would require, the target of the action, the motivation for performing the behaviors, or the 
context in which they occur (Padilla-Walker \& Carlo, 2014). As seen in the existing research cited above, many studies find differential links between correlates of prosocial behaviors and unique forms of behaviors.

The current study utilized a measure that taps into various types, motivations, contexts, and targets of prosocial behaviors. The Prosocial Tendencies Measure (Carlo \& Randall, 2002) was developed to differentiate six forms of prosocial behaviors: dire, compliant, emotional, altruistic, public, and anonymous, and the structure of these six forms of behaviors has been validated in a study with U.S. Mexican adolescents (Carlo et al., 2010). Since then, the measure has been revised to include three potential targets of prosocial behaviors: family, friends, and strangers (adapted from the PTM-R; Carlo et al., 2003).

Three types of prosocial behaviors (dire, compliant, and emotional) have been particularly theoretically linked to U.S. Latino/a culture (Calderón-Tena et al., 2011). Dire prosocial behaviors describe helping in an emergency situation, such as in response to an accident (Carlo \& Randall, 2002). Emotional prosocial behaviors refer to helping in response to an emotionally-evocative situation, such as if someone is hurt and is seeking comfort. Compliant prosocial behaviors occur in response to being asked to help, such as when a parent asks a child to help set the table for dinner (Carlo \& Randall, 2002). For the purposes of the current study, these three types of prosocial behaviors towards three targets will be used.

\section{The Current Study}

The current study uses the literature highlighted above as a backdrop to better understand correlates of prosocial behaviors in a U.S. Latino/a college student sample. 
Adopting Bronfenbrenner's ideas that emphasize the interactions among multiple levels of influence that a person develops within, Figure 1 shows a broad heuristic model that proposes that individual characteristics, relational characteristics, and culture-specific characteristics all interact to affect the individual. The model presented in Figure 1 emphasizes that it is not only one of these domains that can fully explain prosocial behaviors, but that all of these elements fit together like pieces of a puzzle to create a more complete, real-world picture of prosocial behaviors. In addition, the new heuristic model presented in this paper adds a unique contribution to the theoretical literature in that it brings together parts of broader theories, such as Bronfenbrenner's framework, and existing theories on prosocial behaviors to propose a new heuristic model specific to prosocial behaviors that includes bidirectional relations between all of the levels of influence, which is not typically found in current theories on prosocial behaviors.

The current study tests a piece of this heuristic model using one or more variables to represent each level of influence. Figure 2 demonstrates how variables from the current study represent each of the levels of influence presented in the heuristic model. In Figures 3 to 7 , a series of models are presented that were tested in the current study, which focus on individual-level sociocognitive and socioemotive characteristics, a relational characteristic for relationships with mothers and fathers, and the Latino/a cultural value of familism. These models take into account how sociocognitive, socioemotive, and cultural value variables may interact with each other and simultaneously affect prosocial behaviors. The models also account for a relational characteristic, not only for mothers but also for fathers, to examine the interplay of individual-level and relationalcharacteristics as part of the socialization process. 


\section{Chapter 3: Research Questions and Hypotheses}

The current study extends the existing research discussed above by integrating measures of individual-level characteristics (sociocognitive and socioemotive), relational characteristics (maternal and paternal support), and a cultural value (familism) in the same models to test their interrelations, direct relations, and indirect relations to prosocial behaviors. In order to do so, the models were tested using two statistical approaches - $\mathrm{a}$ person-centered approach and a variable-centered approach — to compare the utility of these tests for the study of multiple levels of influence on prosocial behaviors. Four models are presented using a variable-centered approach (two models for type of prosocial behaviors and two models for target of prosocial behaviors; see Appendix C), and one model is used to demonstrate a person-centered approach (see Appendix D). This study was guided by the following primary research questions:

1. Do sociocognitive, socioemotive, relational, and culture-specific variables all directly relate to prosocial behaviors?

2. Do relational characteristics directly relate to sociocognitive, socioemotive, and culture-specific variables?

3. Do relational characteristics indirectly relate to prosocial behaviors via sociocognitive, socioemotive, and culture-specific variables?

4. Can multiple profiles of U.S. Latino/a college students be identified based on similar patterns of socioemotive, sociocognitive, relational, and culturespecific variables?

5. Does profile membership relate to prosocial behaviors? 
Based in the existing theory and research discussed above, the following hypotheses will be used to test the five research questions:

1. Perspective taking, empathic concern, maternal support, paternal support, and familism values will be positively, directly related to prosocial behaviors.

2. Maternal and paternal support will both be positively, directly related to perspective taking, empathic concern, and familism values.

3. Maternal and paternal support will be positively, indirectly related to prosocial behaviors via perspective taking, empathic concern, and familism values, as well as doubly via familism and perspective taking and doubly via perspective taking and empathic concern. There are no a-priori hypotheses about the possibility of significant triple indirect effects from maternal and paternal support to prosocial behaviors via familism, perspective taking, and empathic concern, although these indirect effects may emerge during analyses.

4. Two profiles of individual characteristics (higher empathic concern, perspective taking, familism values, and maternal and paternal support compared to lower empathic concern, perspective taking, familism values, and maternal and paternal support) will be identified, at a minimum. Other groups may emerge but there are no a-priori hypotheses for the other groups.

5. Membership in the high-scoring trait group will be positively related to prosocial behaviors. 


\section{Chapter 4: Research Design and Methods}

\section{Procedure}

The current study is a secondary data analysis of data from the College Health and Adjustment Project led by Dr. Gustavo Carlo and Dr. Sarah Killoren at the University of Missouri. Data was collected from college students at several universities in the United States, including the University of Missouri, University of Connecticut, University of Miami, and Arizona State University. Data was also collected from college students online using MTurk. The aim of this data collection project was to study college students' health and adjustment outcomes. Participants were recruited by classroom and MTurk announcements, and they completed electronic consent forms before participating. Participants completed self-report measures of individual-level correlates of prosocial behaviors, relational correlates of prosocial behaviors, and prosocial behaviors as part of the larger study on college student social behaviors and relationships. All measures were completed in an online survey format and in English. Participation took approximately 45 minutes. Participants on college campuses were compensated by being entered into a raffle for $\$ 10$ gift cards or with extra course credit, and MTurk participants were compensated with $\$ 5$.

\section{Participants}

The participants for the current study were a subsample of the total participants surveyed during data collection. The subsample was determined by selecting the participants in the overall dataset who self-identified as Hispanic/Latino/a in their survey responses. Then, outliers in the age range were eliminated to narrow down the sample to participants between the ages of 18 and 25 years in order to capture only young adult 
college students. After doing so, participants were 250 Latino/a college students between the ages of 18 and 25 ( $M$ age $=21.0, S D=1.9$ years $)$. The gender distribution of the sample was $62.0 \%$ women and $37.6 \%$ men. All participants (100\%) reported being enrolled at four-year universities. The majority of participants (66.8\%) reported English being their native language and that their mothers (84.0\%) and fathers (85.2\%) were also Latina/o. When participants were asked to what Latino/Hispanic group they belong to, the largest subgroup reported identifying as Mexican/Mexican American/Chicano/a (49.6\%). The second largest subgroup in the sample identified as Puerto Rican/Puerto Rican American (26.8\%). In addition, 13.6\% of the sample reported being South American, 6.4\% Dominican/Dominican American, 5.6\% Central American, and 3.6\% Cuban/Cuban American, with 1.2\% of participants reporting Other. The majority of participants (80.8\%) reported being born in the United States.

The majority of participants $(68.0 \%)$ also reported having parents who are married and live together. Parents' educational level varied across the sample. The largest group of participants reported that the highest level of education that their parents completed is high school (29.2\% of mothers and $31.6 \%$ of fathers). A large portion of the sample also reported their parents completing college $(21.6 \%$ of mothers and $22.4 \%$ of fathers). In addition, $18.4 \%$ of participants reported that their mothers completed some college, $14.0 \%$ some high school, $12.4 \%$ graduate/professional school, and $4.0 \%$ were unsure. Further, $15.6 \%$ of participants reported that their fathers completed some high school, $15.2 \%$ some college, $10.4 \%$ graduate/professional school, and $4.8 \%$ were unsure.

\section{Measures}


All measures were electronically completed in self-report format. Measures can be found in Appendix E.

\section{Empathic Concern}

Participants completed a subscale of empathic concern from the Interpersonal Reactivity Index (IRI; Davis, 1983). The IRI is a self-report measure designed to tap into participants' socioemotive and sociocognitive traits, and this measure has been widely used in the study of prosocial behaviors for this purpose. Empathic concern refers to an ability to share the feelings of another person. Participants were asked how well each item describes them. An example item is "I often have tender, concerned feelings for people less fortunate than me." Participants completed the 7 items of the empathic concern subscale and responded on a scale from 0 ("This does not describe me at all") to 5 ("This describes me perfectly"). This empathic concern subscale has been previously used with U.S. Mexican college students (Carlo et al., 2011) and Costa Rican college students (Pierotti et al., 2017) and has demonstrated acceptable reliability. In this sample, the Cronbach's alpha is .88 .

\section{Perspective Taking}

Participants also completed the perspective taking subscale from the IRI (Davis, 1983). Perspective taking describes the ability to consider other people's points of view. Participants were asked to report how well each item describes them. An example item is "I believe that there are two sides to every question and try to look at them both." Participants completed the 7-item subscale on a scale from 0 ("This does not describe me at all") to 5 ("This describes me perfectly"). The perspective taking subscale from the IRI has been used with U.S. Mexican early adolescent samples (Knight et al., 2014) and also 
Costa Rican college students (Pierotti et al., 2017). In this sample, the Cronbach's alpha is .83 .

\section{Familism Values}

To report on their cultural values, participants completed the Mexican American Cultural Values Scale (MACVS; Knight et al., 2010). This measure was designed from discussions with focus groups of U.S. Mexican families about their cultural values, and it has subscales that are intended to capture values from both U.S. Mexican and U.S. mainstream cultures. Familism reflects the cultural value of holding the family in high importance. This measure includes three familism subscales-family emotional support (6 items), family obligations (5 items), and family as referent (5 items) - that were combined into a 16-item composite variable of familism for this study. A study by Knight and colleagues (2014) indicated that because of the strong correlations between the three subscales and evidence that the three subscales are part of a latent construct of familism from the original study (Knight et al., 2010), it is appropriate to combine the three subscales into one familism variable.

In this measure, participants were asked how much they believe each statement. An example item for family emotional support is "Family provides a sense of security because they will always be there for you." An example item for family obligations is "If a relative is having a hard time financially, one should help them out if possible." An example item for family as referent is "When it comes to important decisions, the family should ask for advice from close relatives." Participants responded on a scale from 1 ("Not at all") to 5 ("Completely"). This measure has previously been used with Latino/a adolescents and adults and has shown acceptable reliability (Knight et al., 2014; Knight 
et al., 2010). The construct validity and factor structure of the measure has also been tested and supported (Knight et al., 2010). In this sample, the Cronbach's alpha for the family emotional support subscale is .90 , for the family obligations subscale is .86 , and for the family as a referent subscale is .85 .

\section{Parental Support}

Maternal and paternal support were measured using the support subscale from the Network of Relationships Inventory-Social Provisions Version Short Form (NRI-SPV; Furman \& Buhrmester, 1985). This measure was designed to ask about a variety of relationship characteristics for several important relationships in the person's life. In this measure, support refers to the presence of nurturance, affection, companionship, and reassurance of worth in the relationship (Furman \& Buhrmester, 1985). Participants were asked to reply to each item four times - for their relationship with their mother, father, closest-age sibling, and romantic partner. For the current study, the 7-item support subscale was used and only for relationships with mothers and fathers. Two separate variables were computed, maternal support and paternal support. An example item is “How much does this person help you figure out or fix things?” Participants responded on a scale from 1 ("Little or none") to 5 ("The most"). The NRI has been used with U.S. Latino/a adolescent samples and has shown acceptable reliability (see Stein et al., 2012). In this sample, the Cronbach's alpha for maternal support is .88 and .89 for paternal support.

\section{Prosocial Behaviors}

College students also completed a new adaptation of a well-established measure of multidimensional prosocial behaviors. This measure was adapted from the Prosocial 
Tendencies Measure-Revised (see Carlo et al., 2003; Carlo et al., 2010) in order to include three different targets of prosocial behaviors. Prosocial behaviors broadly refer to actions intended to benefit others (Eisenberg et al., 2006). This 57-item measure assesses six types of prosocial behaviors (dire, emotional, compliant, altruistic, anonymous, and public) towards three different targets (family, friends, and strangers). Participants were asked how well each item applies to their behaviors towards family, friends, and strangers separately. Response options ranged from 1 ("This does not describe me at all") to 5 ("This describes me greatly").

For the current study, this measure was used to represent prosocial behaviors in two different ways: to capture the type of prosocial behaviors or the target of prosocial behaviors, depending on the model (see Figures 3 to 6). For models that investigate the type of prosocial behaviors (Figures 3 and 4), three subscales were used that have been conceptually linked to U.S. Latino/a culture in previous studies with U.S. Mexican early adolescents: dire ( 3 items for each target), emotional (4 items for each target), and compliant ( 2 items for each target; see Calderón-Tena et al., 2011). To do so, the items for all three targets (friends, family, and strangers) were utilized (27 items total). Dire prosocial behaviors refer to helping in emergency situations. An example item is "I tend to help people (i.e., family/friends/strangers) who are in a real crisis or need." Emotional prosocial behaviors describe helping in emotionally-evocative situations. An example item is "It is most fulfilling to me when I can comfort someone (i.e., family/friends/strangers) who is very distressed." Compliant prosocial behaviors denote helping in response to a request. An example item is "When people (i.e., family/friends/strangers) ask me to help them, I do not hesitate.” 
To investigate targets of prosocial behaviors (see Figures 5 and 6), three subscales were calculated using only the dire, emotional, and compliant items but grouping them by target of prosocial behaviors (friends, family, or strangers). Using this method, each subscale results in 9 items. The reliability and validity of this measure has been tested for use with Latino/a samples, including U.S. Mexican early adolescents (Carlo et al., 2010) and Costa Rican young adults (Pierotti et al., 2017). For this sample, the Cronbach's alpha for the dire subscale is .88 , and the Cronbach's alpha for the emotional subscale is 91. The Cronbach's alpha for the compliant subscale is .83. The Cronbach's alpha for prosocial behaviors towards family is .89 and for prosocial behaviors towards friends is .91. The Cronbach's alpha for prosocial behaviors towards strangers is .94 . 


\section{Chapter 5: Data Analysis}

Data preparation and the computation of descriptive statistics was done using SPSS software (IBM Corp., 2016). Two sets of main analyses were conducted: one using a variable-centered approach and one using a person-centered approach. Structural equation modeling (SEM) using path analysis was used for the variable-centered approach, and latent profile analysis (LPA) was used for the person-centered approach. Path analyses and latent profile analyses were conducted with Mplus software (Muthén \& Muthén, 2010). Using SEM path models allows for specific direct and indirect paths among variables to be examined. In contrast, LPA provides unique contributions by being able to examine all five indicator variables simultaneously and to assign participants a group membership based on their scores on the five variables.

\section{Preliminary Analyses}

\section{Data Preparation}

The raw data were prepared by first checking for any data entry errors and outliers. Once the data was cleaned, the study variables were computed following the instructions for each scale. After the variables were created, the data was examined for collinearity, outliers, multivariate normality, and homoscedasticity of residuals following Kline (2011)'s guidelines.

First, to check for collinearity in order to be confident that no two study variables are measuring the same construct, it was necessary to examine the squared multiple correlation between each variable and the other variables (Kline, 2011). If the squared multiple correlation is greater than .90 for a variable, this would indicate multivariate collinearity and the need to potentially eliminate variables from the model or create a 
composite variable. The data were also checked for univariate outliers using $z$ score frequency distributions and box and whisker plots. If any participants' scores were more than three standard deviations from the mean, these would be considered extreme scores that would need to be excluded from further data analysis. The data was also checked for multivariate normality by assessing the skew and kurtosis of each variable. Values for skewness and kurtosis between -2 and +2 are widely considered as acceptable (George \& Mallery, 2010). Lastly, homoscedasticity of residuals was examined using scatterplots and histogram plots of the residuals to ensure that the residuals are distributed uniformly (Kline, 2011). The steps outlined in this paragraph were followed in order to create the cleaned data set with a sample size of 250 participants. As recommended by Kline (2011), it was assumed that missing data is missing at random, because it is unlikely that data are ever missing completely at random. Thus, model-based imputation was used in the statistical tests to provide an estimate for each missing piece of data. In Mplus, this was done using maximum likelihood estimation.

\section{Power Analyses}

In order to determine whether or not there was enough statistical power to conduct the variable-centered models, the rule of 10 participants per parameter to be estimated was used (Kline, 1998). According to this rule, 170 participants would be needed to run each model in Figures 3 through 6 with adequate statistical power. For the use of latent profile analysis, there are not yet set guidelines or conventions for conducting power analyses in the literature (Tein et al., 2013). However, Cohen's $d$ is sometimes used in simulation studies to help determine the utility of model fit indices for latent profile analysis (Tein et al., 2013). Due to the current pandemic, it was not possible 
to access the software needed to calculate Cohen's $d$ at this time; however, the author acknowledges that this would be the appropriate procedure to use in a follow up investigation.

\section{Measurement Reliability}

Cronbach's alphas were calculated for all study variables in order to determine that the variables would be appropriate to propose for the study. According to Kline (2011), alpha levels of .70 or above can be considered acceptable for further analyses.

\section{Descriptive Analyses}

Preliminary analyses were conducted using SPSS. First, descriptive statistics were computed for demographic variables, such as the gender, age, U.S. Latino/a subgroup, and parental education distributions to describe the sample. Then, bivariate correlations between all main study variables, as well as the potential moderating variables of gender and nativity, were calculated in SPSS. See Table 1 for all descriptive statistics and bivariate correlations.

\section{Variable-Centered Analyses}

For the variable-centered approach, path analysis via structural equation modeling using Mplus software (Muthén \& Muthén, 2010) was conducted. Missing data was accounted for using full information likelihood estimation, and bias-corrected bootstrapping was utilized in calculating parameter estimates. The models were evaluated on a range of fit indices. First, whether or not the model is identified was checked by looking at the degrees of freedom for the model (Kline, 2011). If the degrees of freedom were greater than 0 , the model was considered identified, and parameter estimates are reported. Then, a series of model fit indices were evaluated in order to evaluate the 
goodness of fit of the model for the data. One indicator that a model fits the data well is a chi-square statistic that is close to or equal to zero (Kline, 2011). Another index that was used is the Root Mean Square Error of Approximation (RMSEA), which according to Browne and Cudeck (1993) should be less than .08 to indicate reasonable fit. The Comparative Fit Index (CFI) and Tucker Lewis Index (TLI) will also be used, and these should be .95 or greater for good fit (Kline, 2011). Model fit indices for each model are provided in Chapter 6. For models that fit the data well, parameters for each hypothesized direct and indirect path were calculated and are presented using standardized path coefficients.

\section{Person-Centered Analyses}

The person-centered approach utilizes latent profile analysis (LPA) conducted with Mplus software (Muthén \& Muthén, 2010). By using latent profile analysis, profiles of participants who cluster together on certain variables can be identified. For this study, one socioemotive (empathic concern) variable, one sociocognitive (perspective taking) variable, two relational (maternal and paternal support) variables, and one cultural value (familism) variable were used to create profiles.

First, the one-profile solution was tested, and then a series of solutions were tested by adding one profile to each successive test up to five profiles. The best profile solution was determined by analyzing a set of fit indices and statistics. According to Tein and colleagues (2013), a common set of fit indices does not yet exist across the literature, so it is standard to report a combination of indices to evaluate fit as researchers see best. The fit indices that were used in this study are the Bayesian information criterion (BIC), adjusted Bayesian information criterion (ABIC), entropy, Lo-Mendell-Rubin likelihood 
ratio test (LMR), and profile probabilities. See Tables 2, 3, and 4 for LPA fit indices. The best profile solution was determined by the maximum number of profiles that also has an entropy of .80 or above (Muthén \& Muthén, 2010), a significant LMR p-value (Tein et al., 2013), and profile probabilities of .70 or above (Stanley et al., 2017). According to Tein and colleagues (2013), the best-fitting profile solution should also have BIC and $\mathrm{ABIC}$ values that are lower than other profile solutions. Once the final profile solution was identified, profile membership was used to conduct analysis of variance tests in SPSS to investigate relations with types and targets of prosocial behaviors.

\section{Multigroup Analyses}

After bivariate correlations were calculated between the main study variables, gender, nativity, and parents' marital/cohabitation status were considered as possible moderating variables. Gender was significantly correlated with empathic concern, emotional and compliant types of prosocial behaviors, and prosocial behaviors towards family and friends. Nativity was not significantly correlated with any of the main study variables, so it was not included in any further analyses. Parents' marital/cohabitation status was also not significantly correlated with any of the main study variables. In addition, in this sample, the nativity and parents' marital/cohabitation status variables would not provide multiple groups of sufficient size to conduct multigroup analyses using structural equation modeling.

For the variable-centered models, multigroup analyses were conducted for gender in Mplus by testing two competing models: one in which parameters were constrained to be equal across groups (men and women) and one in which parameters were allowed to vary. Then, a Chi-squared difference test was used to test which model fit the data better. 
For all four variable-centered models, a nonsignificant Chi-square indicated that both models fit the data equally well. Thus, the results presented in Chapter 6 are for the entire sample.

For the person-centered models, the possibility of conducting multigroup analyses by gender were considered. Based on the bivariate correlations, empathic concern was the only LPA indicator variable significantly correlated with gender. To further explore this, ANOVAs were conducted in SPSS to see if there were significant group-mean differences by gender on the five LPA indicator variables. As expected, means were significantly different for men and women on empathic concern. However, because there were only significant gender differences on one of five indicator variables and because the sample size in this study when divided by gender would be too small to conduct separate latent profile analyses, multigroup LPA was not further explored. 


\section{Chapter 6: Results}

\section{Descriptive Statistics and Correlations}

Descriptive statistics and bivariate correlations were conducted for the main study variables (see Table 1). Correlations revealed that familism values were positively correlated with empathic concern, maternal support, paternal support, the three forms of prosocial behaviors (dire, emotional, and compliant), and prosocial behaviors towards all three targets (family, friends, and strangers). Empathic concern was positively correlated with perspective taking, maternal support, paternal support, the three forms of prosocial behaviors, and prosocial behaviors towards all three targets. Perspective taking was positively correlated with maternal and paternal support, the three forms of prosocial behaviors, and prosocial behaviors towards all three targets. Maternal support was positively correlated with paternal support, the three forms of prosocial behaviors, and prosocial behaviors towards all three targets. Paternal support was positively correlated with the three forms of prosocial behaviors, prosocial behaviors towards family, and prosocial behaviors towards strangers. All forms and targets of prosocial behaviors were positively correlated with each other form and target of prosocial behaviors.

\section{Results for Variable-Centered Models}

For the variable-centered models, path analysis using structural equation modeling was performed using maximum likelihood estimation with bias-corrected bootstrapping in Mplus.

\section{Maternal Support and Type of Prosocial Behaviors}

Model 1 (see Figure 3) investigated direct and indirect relations between maternal support and three types of prosocial behaviors: dire, emotional, and compliant, as well as 
familism, perspective taking, and empathic concern as intervening variables. The model fit the data well based on a number of fit indices: $\chi^{2}(1)=2.56, n s ; \mathrm{RMSEA}=.08[90 \% \mathrm{CI}$ $.00, .21] ; \mathrm{CFI}=1.00 ; \mathrm{TLI}=.96 ; \mathrm{SRMR}=.02$. Maternal support was directly, positively related to familism, perspective taking, and emotional and compliant prosocial behaviors. Familism was directly, positively related to dire and compliant prosocial behaviors. Perspective taking was directly, positively related to empathic concern, as well as dire, emotional, and compliant prosocial behaviors. Empathic concern was directly, positively related to dire, emotional, and compliant prosocial behaviors.

Testing the potential indirect effects revealed a number of significant indirect effects. Maternal support was positively, indirectly related to dire prosocial behaviors via perspective taking $(\beta=.11, p<.01)$, via familism $(\beta=.08, p<.05)$, and doubly via perspective taking and empathic concern $(\beta=.05, p<.01)$. Maternal support was positively, indirectly related to emotional prosocial behaviors via perspective taking $(\beta=.10, p<.01)$

and doubly via perspective taking and empathic concern $(\beta=.06, p<.01)$. Maternal support was positively, indirectly related to compliant prosocial behaviors via perspective taking $(\beta=.09, p<.01)$, via familism $(\beta=.09, p<.05)$, and doubly via perspective taking and empathic concern $(\beta=.05, p<.05)$. Perspective taking was positively, indirectly related to all three forms of prosocial behaviors via empathic concern: dire $(\beta=.21, p<.001)$, emotional $(\beta=.24, p<.001)$, and compliant $(\beta=.23, p<.001)$.

\section{Paternal Support and Type of Prosocial Behaviors}

Model 2 (see Figure 4) looked at direct and indirect effects from paternal support to dire, emotional, and compliant prosocial behaviors via the intervening mechanisms of familism, perspective taking, and empathic concern. The model fit the data well: 
$\chi^{2}(1)=2.72, n s ; \mathrm{RMSEA}=.08[90 \% \mathrm{CI} .00, .21] ; \mathrm{CFI}=1.00 ; \mathrm{TLI}=.96 ; \mathrm{SRMR}=.02$. Paternal support was directly, positively related to familism. Familism, perspective taking, and empathic concern were all directly, positively related to dire, emotional, and compliant prosocial behaviors.

Paternal support was positively, indirectly related to dire prosocial behaviors via familism $(\beta=.09, p<.05)$. Paternal support was also positively, indirectly related to compliant prosocial behaviors via familism $(\beta=.10, p<.01)$. Perspective taking was positively, indirectly related to all three forms of prosocial behaviors via empathic concern: dire $(\beta=.21, p<.001)$, emotional $(\beta=.25, p<.001)$, and compliant $(\beta=.23, p<.001)$.

\section{Maternal Support and Target of Prosocial Behaviors}

Model 3 (see Figure 5) examined direct and indirect effects from maternal support to prosocial behaviors towards family, friends, and strangers via familism, perspective taking, and empathic concern as intervening variables. The model fit the data well:

$\chi^{2}(1)=2.66, n s ; \mathrm{RMSEA}=.08[90 \% \mathrm{CI} .00, .21] ; \mathrm{CFI}=1.00, \mathrm{TLI}=.95 ; \mathrm{SRMR}=.02$

Maternal support was directly, positively related to familism, perspective taking, and prosocial behaviors towards family and strangers. Familism was directly, positively related to prosocial behaviors towards family and friends. Perspective taking was directly, positively related to empathic concern, as well as prosocial behaviors towards family and strangers. Empathic concern was directly, positively related to family, friends, and strangers.

Maternal support was positively, indirectly related to prosocial behaviors towards family via perspective taking $(\beta=.09, p<.01)$, via familism $(\beta=.10, p<.05)$, and doubly via perspective taking and empathic concern $(\beta=.04, p<.01)$. Maternal support was positively, 
indirectly related to prosocial behaviors towards strangers via perspective taking $(\beta=.10$, $p<.01)$ and doubly via perspective taking and empathic concern $(\beta=.06, p<.01)$. Perspective taking was positively, indirectly related to all three targets of prosocial behaviors via empathic concern: family $(\beta=.18, p<.001)$, friends $(\beta=.21, p<.001)$, and strangers $(\beta=.24, p<.001)$.

\section{Paternal Support and Target of Prosocial Behaviors}

Model 4 (see Figure 6) investigated direct and indirect effects from paternal support to prosocial behaviors towards family, friends, and strangers via the intervening variables of familism, perspective taking, and empathic concern. The model fit the data reasonably well: $\chi^{2}(1)=2.83, n s ; \mathrm{RMSEA}=.09[90 \% \mathrm{CI}=.00, .21]$; $\mathrm{CFI}=1.00$; TLI=.94; $\mathrm{SRMR}=.02$. Paternal support was directly, positively related to familism and prosocial behaviors towards strangers. Familism was directly, positively related to prosocial behaviors towards family and friends. Perspective taking was directly, positively related to empathic concern, as well as to prosocial behaviors towards family, friends, and strangers. Empathic concern was directly, positively related to prosocial behaviors towards family, friends, and strangers.

Paternal support was positively, indirectly related to prosocial behaviors towards family via familism $(\beta=.11, p<.01)$. Paternal support was positively, indirectly related to prosocial behaviors towards friends via familism $(\beta=.10, p<.05)$. Perspective taking was positively, indirectly related to all three targets of prosocial behaviors via empathic

concern: family $(\beta=.19, p<.001)$, friends $(\beta=.22, p<.001)$, and strangers $(\beta=.24, p<.001)$.

\section{Results for Person-Centered Models}

\section{Latent Profile Analysis}


Latent profile analysis was conducted in Mplus to identify the profile solution that best fit the data for the variables of familism, empathic concern, perspective taking, and maternal and paternal support. In order to explore the decision of whether to conduct separate latent profile analyses for maternal and paternal support or to include both in the same model, a series of latent profile analyses were conducted (see Tables 2 to 4 ). First, latent profile analyses were conducted with four variables: familism, empathic concern, perspective taking, and maternal support. See Table 2 for model fit indices for the latent profile analysis including maternal but not paternal support. Then, the same procedure was done but replacing maternal support with paternal support (see Table 3 for model fit indices). Lastly, latent profile analysis was done with all five indicator variables shown in Figure 7 (see Table 4 for model fit indices). Upon evaluating the model fit indices based on the proposed guidelines in Chapter 5 and in thinking about the conceptual model provided in Figure 2, it appeared that 1) it was best to include all five indicator variables in the same latent profile analysis and 2) the four-profile solution fit the data best because the entropy value was above .80, the LMRT $p$-value was significant, and the profile probabilities were all above .70 .

The best fitting solution revealed four profiles. Group 1, the Moderate Scorers $(n=55)$, was characterized by moderate scores on all five indicator variables. Group 2 , the High Individual-Level Variable Scorers ( $n=65)$, was identified by especially elevated scores on empathic concern and perspective taking, as well as more maternal support than paternal support. Group 3, the Low Parental Support Scorers ( $n=9)$, was the smallest group and had low scores on familism and maternal and paternal support but aboveaverage scores on empathic concern and perspective taking. The largest group, Group 4, 
or the High Scorers $(n=121)$, had high scores on all five variables. See Table 5 for group means on indicator and correlate variables.

\section{Analysis of Variance Tests}

Next, a series of analysis of variance tests were conducted using IBM SPSS to compare group means on indicator and correlate variables (see Table 5). Pairwise comparisons were conducted using the Tukey HSD test. The Moderate Scorers had significantly lower scores than the High Individual-Level Variable Scorers on empathic concern and perspective taking, as well as higher scores than the High Individual-Level Variable Scorers on paternal support. The Moderate Scorers' scores were significantly different than the Low Parental Support Scorers' scores and the High Scorers' scores on all indicator variables. The Moderate Scorers' scores were higher than the Low Parental Support Scorers' scores on familism and lower than the Low Parental Support Scorers' scores on empathic concern and perspective taking. The Low Parental Support Scorers' scores were also significantly lower than the Moderate Scorers' scores on maternal and paternal support. The Moderate Scorers were significantly lower than the High Scorers on familism, empathic concern, perspective taking, and maternal and paternal support.

The High Individual-Level Variable Scorers' scores were significantly higher than the Low Parental Support Scorers' scores and significantly lower than the High Scorers' scores on familism. The High Individual-Level Variable Scorers also reported significantly higher scores than the Moderate Scorers on empathic concern and perspective taking. The High Individual-Level Variable Scorers'scores were significantly higher than the Low Parental Support Scorers' scores and lower than the High Scorers' scores on maternal support. For paternal support, the High Individual- 
Level Variable Scorers' scores were significantly lower than the Moderate Scorers' scores and the High Scorers' scores but significantly higher than the Low Parental Support Scorers' scores.

The Low Parental Support Scorers were significantly different than the Moderate Scorers on all variables: lower on familism and maternal and paternal support but higher on empathic concern and perspective taking. The Low Parental Support Scorers were also significantly lower than the High Individual-Level Variable Scorers and the High Scorers on familism and maternal and paternal support. The High Scorers were significantly higher than all other groups (Moderate Scorers, High Individual-Level Variable Scorers, and Low Parental Support Scorers) on familism, maternal support, and paternal support. The High Scorers were also significantly higher than the Moderate Scorers on empathic concern and perspective taking.

In regards to types and targets of prosocial behaviors, significant group differences emerged on a number of variables. When considering type of prosocial behaviors, the High Individual-Level Variable Scorers and the High Scorers both reported scores on dire, emotional, and compliant prosocial behaviors that were significantly higher than the Moderate Scorers. The High Scorers also scored significantly higher than the Low Parental Support Scorers on emotional and compliant prosocial behaviors. In looking at target of prosocial behaviors, the High Individual-Level Variable Scorers and the High Scorers reported significantly higher scores on prosocial behaviors towards family, friends, and strangers than the Moderate Scorers. In addition, the High Individual-Level Variable Scorers and the High Scorers scored significantly higher on prosocial behaviors towards family than the Low Parental Support Scorers. 


\section{Chapter 7: Discussion}

In broad terms, the purpose of this study is to add to the literature on positive development within ethnic-minority families and to do so in a way that includes culturespecific processes, which has been called for by a number of prominent authors in the field (García Coll et al., 1996; McLoyd et al., 2000; Rafaelli et al., 2005). In order to do so, a heuristic model was proposed that was inspired by the work of Bronfenbrenner (1995, 1999), including his bioecological and person-process-context-time models. This heuristic model takes into account macro-level cultural factors, as well as relational and individual variables, that are expected to relate to prosocial behaviors.

Prior theory and research that has informed the study of prosocial behaviors has emphasized the importance of individual-level socioemotional and sociocognitive variables (see Bandura, 1986; Batson, 1998; Hoffman, 2001; Kohlberg, 1976), relationallevel socialization processes (see Grusec et al., 2014), and cultural-level variables (see Calderón-Tena et al., 2011; Carlo \& de Guzman, 2009; Carlo et al., 2014; Knight et al., 1995; Super \& Harkness, 1986; Whiting, 1980). Thus, this study aimed to propose a new way to simultaneously represent all of these pockets of work from the literature on prosocial behaviors.

The goal of this study was to test one iteration of the proposed heuristic model by using one or more variables to represent each level of characteristics that may influence U.S. Latino/a college students' prosocial behaviors. In order to do so, two statistical techniques were used with different purposes: 1) path analysis in order to focus on the relations between variables and 2) latent profile analysis in order to investigate how these variables function simultaneously to identify groups of participants that score differently 
on these variables. The methodology and results of the current study support the use of the proposed heuristic model in order to gain a more detailed understanding of the interplay among these levels of influence on prosocial behaviors.

\section{Variable-Centered Findings}

The purpose of the variable-centered analyses was to investigate the specific relations between sets of variables to provide an understanding of how they operate at the variable level in this sample. Variable-centered analyses were used to test Hypotheses 1 , 2, and 3. Overall, the variable-centered findings lend support to the heuristic model in that significant direct relations between individual-level, relational-level, and culturallevel variables were found.

\section{Hypothesis 1}

Hypothesis 1 predicted that perspective taking, empathic concern, maternal support, paternal support, and familism values would be positively, directly related to prosocial behaviors. Overall, the individual-level sociocognitive and socioemotional variables tended to be positively related to prosocial behaviors, although there were some differences that emerged in the patterns of which variables were related to which forms of prosocial behaviors depending on the model. This partially supports the proposed heuristic model by suggesting that there are relations between the individual-level and cultural-level variables and prosocial behaviors, yet there may be nuances in the ways in which these variables relate.

Maternal Support Models. As expected, perspective taking was positively related to dire, emotional, and compliant prosocial behaviors in the maternal support model. Perspective taking was also positively related to prosocial behaviors towards 
family and strangers in the maternal support model. These findings are consistent with existing theories (e.g., Bandura, 1986; Carlo \& de Guzman, 2009; Carlo et al., 2014) and with findings reported by Carlo and colleagues (2010) and Davis and colleagues (2018) that suggest the importance of perspective taking for prosocial behaviors. As expected, it appears that the ability to see another person's point of view helps U.S. Latino/a college students make decisions about helping others when they are in need of assistance.

However, in the maternal support model, perspective taking was not directly related to prosocial behaviors towards friends. It could be that the way mothers teach their children about perspective taking emphasizes using perspective taking to see the point of view of family and strangers, but not necessarily friends. In the context of friendship, perhaps other variables are more relevant to explaining prosocial behaviors, such as friendship support, reciprocity, or trust. It could be that mothers teach about prosocial behaviors towards friends in a way that emphasizes other components rather than perspective taking, such as empathic concern. Or, it could be that mothers of U.S. Latino/a college students do not often discuss perspective taking and helping towards friends. This finding will be further discussed in the section on Hypothesis 3 regarding the significant indirect effects. Further studies could explore the discourse between mothers and their young adult children about being prosocial towards friends in order to investigate differences in the ways mothers socialize those behaviors.

Empathic concern was positively related to all types and targets of prosocial behaviors in both maternal support models. This finding was expected considering the important emphasis placed on the role of empathic concern in prosocial behaviors that has been discussed in theory and prior research. For example, Hoffman's (2001) 
comprehensive theory of prosocial moral behaviors highlights the importance of empathy when making moral decisions, and Batson's (1998) empathy-altruism hypothesis also emphasizes the role of empathy in prosocial behaviors. Prior research with U.S. Latino/a samples (e.g., Carlo et al., 2011, 2012; Davis et al., 2018) has also found empathic concern to be an important correlate of all forms of prosocial behaviors. Thus, existing research, as well as the results of the present study, continue to support the notion that empathic concern is an important socioemotive trait that is evoked in opportunities for prosocial behaviors. The results of the current study suggest that U.S. Latino/a college students use these feelings of care and concern for other people that become salient as a motivation for all forms of prosocial behaviors when opportunities to help arise.

The cultural-level variable, familism values, was positively related to dire prosocial behaviors, as well as prosocial behaviors towards family and friends, in the maternal support models. Knight and colleagues' (1995) cultural value transmission model suggests that cultural values, often learned from parents, play an important part in acting prosocially. In a test of Knight and colleagues' (1995) cultural value transmission model, Calderón-Tena and colleagues (2011) found familism values to be positively related to a composite of prosocial behaviors for U.S. Mexican early adolescents. Based on this and other existing research, it was expected that familism values would be positively related to prosocial behaviors in the current sample, but there were no specific predictions about which forms. It is possible that familism values are positively related to dire prosocial behaviors because familism encourages considering the needs of others, which may extend to helping any other person who is in an emergency. As predicted, familism values were positively related to prosocial behaviors towards family and 
friends, likely because endorsing a value that emphasizes the importance of family theoretically should be related to helping family more. In turn, this value endorsement may extend beyond the family to friends. Friends, especially for college students, may play a role very similar to family if students are spending more time away from home (or potentially living away from home and with their friends) and spending a lot of time in close, intimate friend groups.

In regards to relational-level variables, maternal support was directly, positively related to emotional and compliant prosocial behaviors but not dire prosocial behaviors. Maternal support was also directly, positively related to prosocial behaviors towards family and strangers but not towards friends. These findings raise interesting questions about the socialization of prosocial behaviors happening within mother-child relationships. In Grusec and Goodnow's (1994) model of value internalization, the authors discussed that value internalization is more likely to occur within a positive parent-child relationship. Thus, it was expected that maternal support would be a correlate of all forms of prosocial behaviors. In Davis and colleagues' (2015) study with an early adolescent U.S. Mexican sample, maternal support was positively related to dire and compliant prosocial behaviors but not emotional. It could be that U.S. Latino/a college students especially rely on their mothers' support in and learn from their mothers about how they make decisions about emotional and compliant prosocial behaviors. Mothers in U.S. Latino/a families may play an important role in the family as the nurturers who provide emotional comfort and who, in addition, expect compliance when requesting help. These also may be the prosocial behaviors that U.S. Latino/a college students observe their mothers conducting the most, so they may internalize the 
importance of those types of prosocial behaviors within the mother-child relationship context and later imitate the behaviors that they observe in accordance with social cognitive theory (Bandura, 1986).

Concerning the finding that maternal support was not related to dire prosocial behaviors in the current study, it could be that there are age-related differences in the types of prosocial behaviors that are related to maternal support. For instance, perhaps at a younger age, such as in Davis and colleagues' (2015) study, maternal support is more needed in order to participate in dire prosocial behaviors. By young adulthood, dire prosocial behaviors may be less related to a relational-level variable and more related to an individual-level variable, such as one's own internalized moral values and identity. In regards to the target of prosocial behaviors, it is possible that mothers emphasize prosocial behaviors towards family and strangers more than prosocial behaviors towards friends in their socialization processes or in the developmental affordances that they offer their young adult children. Instead, U.S. Latino/a college students may learn about prosocial behaviors towards friends in other contexts, such as within the friend group via friendship support.

Paternal Support Models. In the paternal support models, perspective taking was also positively related to dire, emotional, and compliant prosocial behaviors, as well as prosocial behaviors towards family, friends, and strangers. These findings were expected based on existing theories (e.g., Bandura, 1986; Carlo \& de Guzman, 2009; Carlo et al., 2014) and with prior empirical findings by Carlo and colleagues (2010) and Davis and colleagues (2018) emphasizing the role of perspective taking as a correlate of unique types of prosocial behaviors. It was also expected that perspective taking would 
be necessary for all targets of prosocial behaviors. Thus, it appears that U.S. Latino/a college students who score highly on perspective taking apply that trait to situations when making decisions about whether to help all recipients.

Empathic concern was also positively related to all types and targets of prosocial behaviors in the paternal support models. As discussed above in the section on maternal support, these findings are consistent with prior theory and research on empathic concern that emphasizes the role of empathic concern as a motivator for prosocial behaviors (see Batson, 1998; Carlo et al., 2011, 2012; Davis et al., 2018; Hoffman, 2001). It is likely that U.S. Latino/a college students are motivated by these feelings of care and concern for others when they witness an opportunity to help and that this social emotion leads them to act, regardless of the type of behavior needed or the person in need of assistance.

As found in the maternal support models, familism values were positively related to dire prosocial behaviors, as well as prosocial behaviors towards family and friends, in the paternal support models. Familism values were also positively related to emotional and compliant prosocial behaviors only in the paternal support model. These findings were expected and align with prior theory and research discussed above in the section on maternal support (see Calderón-Tena et al., 2011; Knight et al., 1995). The findings that familism values were also positively related to emotional and compliant prosocial behaviors only in the paternal support model was unexpected. It could be that in U.S. Latino/a families, fathers play an especially important role in their children's lives. The father may be seen has the head of the household; thus, children may learn that valuing the family involves complying with the father's requests for help when asked. In addition, as the head of the household the father may be seen as the person who children 
turn to when in distress, so the connection between familism values and emotional prosocial behaviors may be due to the children learning from the father that part of valuing the family involves resolving distress when needed. This finding is inconsistent with a prior longitudinal study conducted with U.S. Mexican adolescents, which found mothers', but not fathers' ethnic socialization practices to predict adolescents' ethnic identity and in turn, prosocial behaviors (Knight et al., 2016). Knight and colleagues (2016) found fathers' own familism values to be predictive of their children's familism values, which in turn were related to prosocial behaviors, but there were no indirect effects from fathers' familism values or ethnic socialization practices to their children's prosocial behaviors (Knight et al., 2016). One potential explanation for this discrepancy could be the age differences between the participants in Knight and colleagues' (2016) study compared to the current study. Or, this could be due to the fact that the participants in the current study, who are all enrolled in college, are a unique subset of all U.S. Latino/a young adults. It is also possible that by young adulthood, fathers may talk about and/or demonstrate familism values in ways within their families that their young adult children connect more with emotional and compliant forms of prosocial behaviors than other forms of prosocial behaviors and that they later imitate (see Bandura, 1986). Future studies that examine how fathers of young adult children socialize familism and unique forms of prosocial behaviors could be helpful in understanding how fathers teach these concepts.

Interestingly, paternal support was only positively related to one target of prosocial behaviors: prosocial behaviors towards strangers. Based on Grusec and Goodnow's (1994) model of value internalization, it was expected that paternal support 
would also have been positively related to all types and targets of prosocial behaviors. However, there was no prior research available to aid in making more specific predictions about targets of prosocial behaviors. It could be the case that through their socialization practices and messages, fathers in this sample taught messages about acting prosocially towards strangers more so than other targets of prosocial behaviors. It is possible that within the U.S. Latino/a immigrant family context, fathers, who are often seen as the head of the family, socialize their young adult children to help strangers in the receiving community as a way to encourage their children to protect themselves by fitting in with the mainstream culture and respecting authority figures. It could be that within families that have both a mother and a father that the father takes on the role of socializing messages about how to interact with strangers in the receiving culture to promote the safety of oneself and one's family and that mothers, more so than fathers, are the ones to be more involved in socializing prosocial behaviors towards close relationships, such as family and friends.

Overall, Hypothesis 1, which predicted that perspective taking, empathic concern, maternal support, paternal support, and familism values would be positively, directly related to prosocial behaviors, was partially supported. Some nuanced findings emerged that suggest that these relations may differ depending on the relational context being taken into account. Future research should further explore the roles of fathers and mothers in U.S. Latino/a college students' lives to better understand how these relationships differently affect prosocial behaviors.

\section{Hypothesis 2}


Hypothesis 2 predicted that maternal and paternal support would both be positively, directly related to familism values, perspective taking, and empathic concern. Overall, these hypotheses were partially supported, which will be further discussed in the sections on maternal and paternal support separately. These hypotheses were fully supported for relations between both maternal and paternal support and familism values. These hypotheses were also supported in relations between maternal support and perspective taking. Overall, these results lend support to the proposed heuristic model by demonstrating that there are interactions between correlates of prosocial behaviors at the relational and individual levels.

Maternal Support Models. As predicted, maternal support was directly, positively related to familism values in both maternal support models. These results were expected, because according to Grusec and Goodnow's (1994) model of value internalization, positive relationship qualities foster the internalization of values; thus, this line of thinking likely extends to the internalization of cultural values as well. This is consistent with research done in Mexico that found that parental warmth and support was related to children's positive feelings towards their family (Bronstein, 1994). Especially because familism values directly relate to the family context, it was expected that the more supportive parents are, the more likely their children are to endorse the importance of family. For the current sample, it seems that U.S. Latino/a college students who perceived receiving support from their mothers did tend to internalize familism values.

The expected link between maternal support and perspective taking was also supported. Considering that this effect was found in the maternal support models but not the paternal support models implies that there could be interesting variations in the 
parent-child interactions like the content of the discourse or the opportunities provided to develop perspective taking, such as the developmental affordances discussed by Calderón-Tena and colleagues (2011). Perhaps mothers, rather than fathers, are more likely to use parental inductions, which have been linked to sympathy (a combination of both perspective taking and empathic concern), in a study with Columbian adolescents and parents (see Guevara et al., 2015). Although no prior studies on parental support and perspective taking were found in the literature for U.S. Latino/a college student samples, studies on other indicators of positive parenting were positively related to perspective taking (e.g., Shen et al., 2013). Shen and colleagues (2013) found maternal induction to be positively related to perspective taking for a U.S. Mexican early adolescent subsample of their study. Within U.S. Latino/a families, mothers may be seen as nurturers and may engage in that role by having thoughtful, deep discussions with their children about moral issues. Thus, mothers may be more likely to teach their children about perspective taking due to traditional gender roles often endorsed as a U.S. Latino/a cultural value. In this case, mothers may be seen, and may see themselves, and the parent responsible for providing nurturance via conversation and teaching their children how to understand and care for others. This emphasizes an important point that a supportive relationship may or may not be enough on its own; instead, it is critical to consider the content and quality of the discourse occurring within those relationships. Future studies could investigate maternal discourse content and quality in order to see if those factors help to explain why support was only related to perspective taking for mothers in this sample.

Hypothesis 2 was not supported for relations between maternal support and empathic concern, which was not expected. In a prior study with U.S. Mexican college 
students, researchers found closest parent attachment to be positively related to empathic concern (Carlo et al., 2012). As will be discussed next with Hypothesis 3, even though there were not significant direct relations, there was a significant indirect effect of maternal support on empathic concern via perspective taking in this sample. Perhaps the direct influence of maternal support on empathic concern is not strong enough to be consistent across samples, but rather the importance of mothers' support is seen in how it helps foster perspective taking, which in turn contributes to empathic concern.

Paternal Support Models. As found in the maternal support models, paternal support was also positively related to familism values in the paternal support models. This is again consistent with models of value internalization (Grusec \& Goodnow, 1994) that emphasize that positive parent-child relationship qualities are likely to foster the internalization of values. This was expected especially for familism values, because these values directly relate to the family context. Fathers who are supportive of their young adult children may demonstrate a strong, supportive presence in the family context and therefore would be likely to teach their children about the importance of family via modeling, in accordance with social cognitive theory (Bandura, 1986), that their children may then internalize.

The expected link between paternal support and perspective taking was not supported as it was for mothers. It could be that the ways in which U.S. Latino/a college students' fathers converse with them do not foster thinking about other people's point of view or offer opportunities to practice doing so within those conversations to the same extent that mothers do. For example, if the father is seen as the head of the household who rules with authority, the conversations between father and child may be more direct 
and authoritarian, placing less emphasis on taking the perspective of others and more emphasis on abiding to requests or rules. Or, even fathers who are supportive may not provide developmental affordances for perspective taking in the same way that mothers do and instead may focus on teaching their young adult children about other concepts based on traditional gender roles.

Hypothesis 2 was also not supported for relations between paternal support and empathic concern, which was not expected. For fathers, it is possible that paternal support was not directly related to empathic concern because the socialization processes, such as discourse, and messages that fathers use may be different than the socialization processes and messages that mothers use. For example, these results may stem from the influence of traditional gender roles in U.S. Latino/a culture, which would suggest that mothers operate as the nurturers who would be more likely to teach about care, compassion, and feeling empathy for others. In contrast, fathers, seen as more of the provider and head of the household and less in a nurturing role, may not talk to or teach their children in ways that focus on teaching care and concern for others.

Taken together, the results of the current study partially support Hypothesis 2, especially for familism values and for perspective taking when considering the motherchild relationship. It appears that there are nuances in how relational-level variables relate to individual-level variables. Therefore, moving forward it will be important to continue to study a variety of relationships and relational-level variables to gain a better understanding of how each set of relational-level and individual-level variables interact with each other. In general, these findings provide partial support for the proposed 
heuristic model that suggests that relational-level variables relate to both individual-level and cultural-level variables.

\section{Hypothesis 3}

Hypothesis 3 posited that maternal and paternal support would be positively, indirectly related to prosocial behaviors via perspective taking, empathic concern, and familism values. These hypotheses also extended to potential double indirect effects via familism and perspective taking and via perspective taking and empathic concern. These hypotheses were based in ideas from the socialization literature (e.g., Grusec et al., 2014; Grusec \& Goodnow, 1994) that suggest that the parent-child relationship is an important domain for teaching values. In addition, Knight and colleagues' (1995) cultural value transmission model emphasizes the role of parents and their values and practices in promoting children's cultural values and prosocial behaviors. Taken together with other theories that discuss the importance of sociocognitive and socioemotive processes in prosocial behaviors (e.g., Bandura, 1986; Carlo \& de Guzman, 2009; Carlo et al., 2014; Hoffman, 2001), it corresponds that these pieces to fit together such that the sociocognitive and socioemotive processes could act as intervening mechanisms in the relations between socialization variables and prosocial behaviors.

Overall, the results showed partial support for the hypotheses that maternal and paternal support would be indirectly related to prosocial behaviors via the intervening mechanisms of perspective taking, empathic concern, and familism values. These findings suggest that in some, but not all cases, sociocognitive and socioemotive processes measured at the individual level work together as intervening mechanisms between relational-level variables and prosocial behaviors. This partially supports 
Feshbach's (1987) proposition that people are more likely to be prosocial when they have both sociocognitive and socioemotive traits, rather than one or the other. These findings also provide partial support for the heuristic model proposed in Figure 1, which posits that relational-level variables interact with individual-level variables in relation to prosocial behaviors. In addition, it appears that variables interact with each other within each level of influence in the heuristic model; more specifically, sociocognitive and socioemotive traits seem to interact at the individual level to influence prosocial behaviors.

Maternal Support Models. Maternal support was indirectly related to dire and compliant prosocial behaviors and prosocial behaviors towards family via familism values. These findings are consistent with the Knight and colleagues' (1995) cultural value transmission model, which theorizes that parenting practices should be positively related to prosocial behaviors via adolescents' own adoption of cultural values. Knight and colleagues' (2016) longitudinal study found maternal parenting variables (mothers' familism values and mother's ethnic socialization practices) to be indirectly related to both dire and compliant prosocial behaviors via familism values. Thus, it appears that one way that maternal support encourages prosocial behaviors, or these types at least, is via promoting familism values. It is possible that within these supportive relationships, children are provided with the developmental affordances (see Calderón-Tena et al., 2011) to learn about the importance of family and of helping in emergency situations or when asked. In the case of dire prosocial behaviors, it could be that part of the teachings of familism values that young adult children learn from their supportive mothers involves being ready to help when emergencies arise. Even though opportunities for dire prosocial 
behaviors may not always emerge in the family context, there may be opportunities from time to time to help other family members in an emergency. Thus, this sense of helping others in an emergency that one learns within the mother-child relationship may also extend to helping any other person in an emergency situation. In regards to compliant prosocial behaviors, these behaviors likely often take place within the family context and have occurred for U.S. Latino/a college students' entire lives in the family unit. Mothers who are supportive appear to teach their young adult children to see the importance of helping family members when asked as part of familism values; thus, they may teach that part of valuing the family means complying with requests.

In addition, maternal support was indirectly related to dire, emotional, and compliant prosocial behaviors, as well as prosocial behaviors towards family and strangers, via perspective taking and doubly via perspective taking and empathic concern. Overall, it appears that a supportive mother-child relationship may be an important context for the development of sociocognitive and socioemotive processes, which are both important antecedents of prosocial behaviors (Carlo \& de Guzman, 2009). Prior research and theory have also suggested that perspective taking is a necessary component of empathic concern (see Eisenberg, 1991), and another recent study with U.S. Latino/a adolescents supported this notion (Davis et al., 2018). Similarly, Feshbach (1987) suggested that people are more likely to be prosocial when they have both the sociocognitive and the socioemotive processes, rather than one or the other. Therefore, the double indirect effect in which both perspective taking and empathic concern act as intervening mechanisms in this relation is especially interesting in that it accounts for perspective taking as an important sociocognitive component of empathic concern and 
supports these theoretical views. It seems as though U.S. Latino/a college students who perceive support from their mothers seem to internalize the perspective taking and empathic concern that their mothers help foster and teach them, and those traits then help foster helping behaviors in opportunities to help almost consistently across types and targets of prosocial behaviors. However, these indirect effects were not found for prosocial behaviors towards friends. Instead, it is likely that other variables, such as perceived support from friends or belongingness to the friend group, become more relevant in the friendship context.

Paternal Support Models. As found in the maternal support models, paternal support was also indirectly related to dire and compliant prosocial behaviors and prosocial behaviors towards family via familism values. As discussed in the maternal support section, this is consistent with Knight and colleagues' (1995) cultural value transmission model such that parenting variables are related to prosocial behaviors via their effect on children's own value endorsement. It could be that fathers who are supportive provide their young adult children with developmental affordances (see Calderón-Tena et al., 2011) that promote familism values, and then these familism values are related to dire and compliant prosocial behaviors because those prosocial behaviors are encouraged in the family context. As was discussed for these same indirect effects for maternal support, it could be that supportive fathers teach their children that part of familism is being ready to help if an emergency arises. Acknowledging that dire prosocial behaviors may not often or always occur in the family context, it is still likely that occasionally they do. This duty to help others in an emergency situation may be taught about in such a way that U.S. Latino/a college students extend it to a duty to help any 
other people in an emergency. For compliant prosocial behaviors, fathers may teach their young adult children that part of familism is a duty to comply to the needs of the family when requested. It could be that U.S. Latino/a college students also call upon these values when any other person asks for help, not only family members.

In contrast to the maternal support model, no indirect effects were found from paternal support to prosocial behaviors via perspective taking or doubly via perspective taking and empathic concern. It could be that in the father-child relationship, where the father may be seen as the head of the household, fathers' support affects prosocial behaviors more through familism values, whereby they teach their children to care for others because it is seen as a duty rather than by emphasizing taking the perspective of others and understanding their point of view. Interestingly, paternal support was indirectly related to prosocial behaviors towards friends via familism values, even though paternal support was not directly related to prosocial behaviors towards friends. As discussed in Hypothesis 1, it is possible that there is something unique about the socialization that occurs within the father-child relationship that strengthened the relation between familism values and prosocial behaviors towards friends only for the paternal model. It could be that fathers teach their young adult children about family and friends in a way that is different than mothers, and this could affect their young adult children's prosocial behaviors towards friends. For example, for college students, friends may almost be seen as part of the family, because college students are likely to spend a lot of time with their friends and may even live with them at college. It is possible that fathers who teach their young adult children about familism extend the radius of care to include 
friends. As mentioned above, a future line of research could investigate the discourse in parent-young adult child relationships about who "counts" as family.

Taken together, the variable-centered findings provided partial support for the proposed heuristic model by demonstrating that the variables used to represent the individual, relational, and cultural levels of influence do relate to each other and in unique ways. Next, the person-centered findings can be used to help understand how these relations among the correlates of prosocial behaviors included in this study simultaneously interact with each other in relation to prosocial behaviors.

\section{Person-Centered Findings}

The person-centered findings supported the proposed heuristic model that suggests that variables at all three levels of influence interact simultaneously to relate to prosocial behaviors. Results supported the utility of this approach in order to identify unique profiles that reveal how these variables work together in different ways, which would have been impossible to determine from the variable-centered analyses. Personcentered analyses were used to test Hypotheses 4 and 5.

\section{Hypothesis 4}

Hypothesis 4 suggested that two profiles of individual characteristics (higher scores on individual and relational variables compared to lower scores on individual and relational variables) would be identified, at a minimum. The latent profile analysis revealed that the best-fitting solution identified four groups, and the existence of these groups both support and extend Hypothesis 4. As expected, there was a group that scored higher than other groups on all variables (the High Scorers). The High Scorers group, which was the largest group, had high scores on all individual (empathic concern and 
perspective taking), relational (maternal and paternal support), and cultural-level (familism) variables. A group also emerged that scored lower than other groups on all variables, the Moderate Scorers group, yet this group still demonstrated moderate scores on all variables. The Moderate Scorers group was characterized by moderate scores on all five indicator variables: familism, empathic concern, perspective taking, maternal support, and paternal support. In addition, two other groups emerged in the analyses. The High Individual-Level Variable Scorers group had high scores on individual-level variables (empathic concern and perspective taking) but moderate scores on relational (maternal and paternal support) and cultural-level (familism) variables. The Low Parental Support Scorers group, which was the smallest group, had high scores on individual-level variables (empathic concern and perspective taking) but very low scores on relational (maternal and paternal support) and cultural-level (familism) variables.

Because prior theory and existing research emphasizes the importance of individual-level sociocognitive and socioemotive variables (e.g., Batson, 1998; Feshbach, 1987; Hoffman, 2001), relational-level variables (e.g., Grusec et al., 2014), and culturallevel variables (e.g., Carlo et al., 2014; Knight et al., 1995; Super \& Harkness, 1986; Whiting, 1980) in prosocial behaviors, it was expected that a group would emerge that scored relatively high in all of these areas. This group, the High Scorers group ( $n=121)$, was the largest group identified. This means that the largest group in the sample reported having these sociocognitive and socioemotive traits, receiving support from both their mother and father, and endorsing the cultural value of familism. Considering that much of the literature, especially the literature on U.S. Latino/a families, has been dominated by a deficit approach and studying delinquent outcomes in the past (García Coll et al., 
1996), this is an important finding in itself. The majority of participants in this sample of U.S. Latino/a college students were, in fact, doing quite well at all three of these levels of influence, which was expected considering U.S. Latino/a young adults who are in college tend to be rather well-adjusted. This group also demonstrates that all five of these correlates of prosocial behaviors work together simultaneously to promote prosocial behaviors, as was predicted by the proposed heuristic model.

The Moderate Scorers group $(n=55)$ scored below the total sample mean on all five indicator variables, yet their scores could still be considered moderate on all five variables. None of their scores especially stood out compared to the other groups, and their scores on maternal and paternal support were quite similar to each other. Thus, this could be seen as a moderate group. It was expected that there would be a group in the population that scored lower on the indicator variables, and it is interesting that the "low" group resulted to still be reporting moderate scores on all of these variables. This again can be considered an important finding that the lowest group on these important sociocognitive and socioemotive variables was still scoring moderately on the scales. However, it is important to acknowledge that the entire sample is U.S. Latino/as who are in college, meaning that this is typically a well-adjusted group, and these results may not generalize to the entire Latino/a young adult population.

The High Individual-Level Variable Scorers group $(n=65)$ scored especially high on the individual-level variables, and in fact, this group had the highest scores on both empathic concern and perspective taking out of all four groups. Their scores on the relational variables and the cultural-level variable of familism were moderate. This group's scores on paternal support were quite lower than their scores on maternal 
support. Thus, participants in this group may be especially experiencing support within the mother-child relationship but perhaps not as much in the father-child relationship. Theoretically, it makes sense that in the real world that there would be segments of the population that have more support from one caregiver than the other. This leads to interesting questions about how socialization processes look within the family context when one parent is supportive and the other parent is not as supportive. Future studies could explore these relationship dynamics in the family context and their effects on individual development.

The Low Parental Support Scorers group $(n=9)$ was a particularly interesting and valuable finding. While the latent profile analysis identified this as a unique group, the smallest number of participants fell into this group. However, this group was kept for analysis and interpretation, because it has important theoretical and real-world implications. The Low Parental Support Scorers reported low scores on both maternal and paternal support, which likely does occur in the real world in some cases. Yet what is incredibly interesting is that despite low levels of parental support, this group scored significantly higher than Moderate Scorers on empathic concern and perspective taking. Therefore, it seems that even without the influence of maternal and paternal support in these participants' lives, they developed strong individual-level sociocognitive and socioemotive traits. This group, and these findings, raise the question of whether other socialization domains and/or biological processes related to prosocial behaviors become more important contexts for acquiring these traits when parental support is not present. Future studies could focus on developing models specific to this group to better 
understand what other contexts of development could be bolstered by interventions in order to compensate for the lack of parental support.

\section{Hypothesis 5}

Hypothesis 5 was that membership in the high-scoring group would be positively related to prosocial behaviors. The results of the latent profile analysis support this hypothesis that membership in groups that scored higher on the individual, relational, and cultural variables than the other groups also scored higher on prosocial behaviors than the other groups. The High Scorers group, which had high scores on all variables, scored significantly higher than the Moderate Scorers group on dire, emotional, and compliant prosocial behaviors, as well as prosocial behaviors towards family, friends, and strangers. It was expected that higher scores on maternal support, paternal support, familism, empathic concern, and perspective taking would be related to more prosocial behaviors, and membership in the High Scorers group was indeed related to more of every type and target of prosocial behaviors. This provides support for the heuristic model that aims to integrate various pieces of the existing theories and literature on prosocial behaviors. This suggests that all of these levels of influence — individual, relational, and cultural—do relate to prosocial behaviors, and scoring highly at all of these levels is related to the highest scores on all forms of prosocial behaviors.

The High Scorers group also scored significantly higher than the Low Support Scorers group on emotional and compliant prosocial behaviors and prosocial behaviors towards family. Considering that Low Parental Support Scorers experienced lower levels of parental support and reported lower familism endorsement than High Scorers, it was expected that they would not be engaging in as many prosocial behaviors towards family. 
Future studies could explore the mechanisms by which this occurs or the contextual factors and/or life events that would lead to a small number of U.S. Latino/as perceiving low levels of support from their parents. Lower scores on emotional and compliant prosocial behaviors could be explained by these types of prosocial behaviors being especially likely to happen and/or to be socialized within the family context. For example, the path models revealed significant indirect effects for parental support to compliant prosocial behaviors via familism values. Thus, it could be that the socialization of familism in the family context is an important process by which compliant prosocial behaviors are taught. Similarly, the family context may typically provide many opportunities, or developmental affordances (see Calderón-Tena et al., 2011), to engage in emotional prosocial behaviors. However, in families where there is not much perceived parental support, children may either 1) not be given these opportunities to the same extent as in families with higher levels of parental support or 2) children may choose not to engage in those opportunities to comfort others if they do not feel that they receive that same type of support from their parents.

High Individual-Level Variable Scorers also scored significantly higher than Moderate Scorers on all forms of prosocial behaviors: dire, emotional, and compliant types, as well as prosocial behaviors towards family, friends, and strangers. High Individual-Level Variable Scorers reported the highest scores on empathic concern and perspective taking out of all the groups but moderate scores on relational and cultural variables. Interestingly, even though High Individual-Level Variable Scorers scored lower than Moderate Scorers on paternal support, this did not seem to matter in relation to prosocial behaviors when all five variables were taken into account simultaneously. 
Thus, it appears that the other variables could in fact be compensating for this lower level of paternal support. So, even though Moderate Scorers had higher scores on paternal support, High Individual-Level Variable Scorers scored significantly higher on all measures of prosocial behaviors. By only using path models, this constellation of variables would not have been identified. Thus, future studies could explore the means by which other variables at the individual, relational (in other relationships), or cultural level could compensate for a low level on one of the other variables.

High Individual-Level Variable Scorers also scored significantly higher than Low Parental Support Scorers on prosocial behaviors towards family. This is not astounding due to the fact that High Individual-Level Variable Scorers reported high scores on both familism values and maternal support and that Low Parental Support Scorers scored low on all variables related to the family context. However, it is interesting that with even with low levels of paternal support, High Individual-Level Variable Scorers still scored significantly higher than Low Parental Support Scorers. This could further support the idea of investigating how other variables work as compensatory mechanisms for each other when one variable is low.

For example, perhaps High Individual-Level Variable Scorers had higher scores than Low Parental Support Scorers on prosocial behaviors towards family because High Individual-Level Variable Scorers were still experiencing support in the family context from maternal support and endorsing the importance of family. Thus, perhaps when one family-related variable is low out of three, this does not drastically affect prosocial behaviors towards family for this group, but when all three family-related variables are low, it does. This finding suggests the need to further explore this heuristic model with 
additional family-related variables to better understand how various elements of the family context could compensate for low scores on one or more family-related variables. For example, if sibling support was added to the latent profile analysis, it may be possible to identify a group that scores low on parental variables yet has high sibling support. It would be interesting to investigate how this would relate to overall prosocial behaviors towards family in that case. Or, it could be possible that there is some sort of threshold for how many variables are low before prosocial behaviors is affected. For example, maybe it is still possible to score higher than other groups on prosocial behaviors towards family when one or two variables are low, but perhaps three variables act as some sort of threshold. These are questions that would need to be investigated in future studies with more measures on the family context. 


\section{Chapter 8: Overall Strengths, Limitations, Conclusions, and Future Directions}

This section will review perceived strengths, limitations, and general conclusions from the present study. The chapter will close with a discussion of future directions and ideas generated by the proposed heuristic model and results of the current study.

\section{Strengths of the Current Study}

The current study has several strengths that warrant discussion before considering the limitations. One strength is the sampling strategy. While most of the existing literature on U.S. Latino/as has focused on participants of Mexican-origin, the purposive sampling strategy used in this study provides representation from more U.S. Latino/a subgroups. This is important, because each Latino/a subgroup has different historical and contextual experiences when coming to the United States (Fuligni \& Perreira, 2009). Future studies that aim to make generalizations about the population of U.S. Latino/a college students should continue to represent each subgroup and in appropriate proportions in order to be more illustrative of the actual population of U.S. Latino/a college students. However, more studies are needed that focus solely on one Latino/a subgroup at a time in order to create models specific to the experiences of each subgroup. Although the current study included data from various Latino/a subgroups, important subgroup differences may have also been overlooked due to analyzing the data as one sample, which will be discussed below with the limitations.

Another strength of this study is the use of two unique statistical techniques in order to better understand the functions of the variables of interest in this sample. Using path analysis, which has traditionally been most visible in the literature on prosocial behaviors, provides a foundational understanding of how variables relate to one another. 
However, only using path analysis would fail to capture the ways in which these variables simultaneously interact on a daily basis in the lives of individual people. Therefore, the intention of also using latent profile analysis was to investigate how a set of variables that tap into each of the levels presented in the heuristic model function together simultaneously, yet differently, for various groups of people within the sample. For example, the results revealed that groups of participants do score differently at all three levels of influence and in different patterns. By making variable-centered conclusions about the importance of maternal and paternal support for prosocial behaviors, researchers are not capturing what is happening for the group of participants who do not perceive high levels of maternal or paternal support. Yet this group reported high scores on empathic concern and perspective taking at the individual level, and being in this group was related to high levels of prosocial behaviors. These differences would not be recognized if it were not for conducting person-centered analyses to further investigate the variable-centered models.

In addition, this study further explored the multidimensionality of prosocial behaviors by looking at not only type of prosocial behaviors but also target. The field of prosocial behaviors has, thankfully, moved towards a better understanding of the fact that prosocial behaviors are quite multidimensional. For a few decades now, work has been done to better understand different types of prosocial behaviors and the ways in which these types may have different correlates. There has been growth in that direction in the literature, although as always, more research is needed to keep adding to this knowledge on type of prosocial behaviors. In recent years, there has also been an increasing interest in better understanding the target of the prosocial behaviors, which is important 
considering that by nature prosocial behaviors have a social component and likely involve at least two people in the action. Thus, in order to understand motivations for the behaviors more completely, it is important to take into account the recipient of the action and why a person may be more or less likely to help based on the relationship. This study helps to address some of those questions by investigating whether the target of prosocial behaviors are family, friends, or strangers. Future studies could dive even deeper into understanding characteristics of the target and the relationship between the target and the actor.

Lastly, this study included a variable capturing both maternal and paternal relationship qualities, helping the field to move beyond a bias towards research on mothers only. Future studies using this conceptual model could include more relationships from the individuals' microsystem, such as siblings, romantic partners, friends, grandparents, teachers, and other influential relational figures. Although this study had a number of strengths that set it apart from the existing literature, it is important to next discuss the limitations to this work.

\section{Limitations of the Current Study}

Although this study has many strengths and makes unique contributions to the literature, there are always limitations to be expected of any study. First, there were some limitations in the types of analyses used. This study was a correlational design; thus, it is impossible to determine cause and effect relations among variables. This limits the amount of confidence that one can have in the direction effects, because the direction of effects could in reality be the opposite of the proposed directions in the models. It must also be noted that the data were collected in self-report format from only one informant, 
thus affecting the shared (or common) method variance. Because only one person was rating each of the constructs of interest in this study, the variance being accounted for by these variables may be affected by this measurement technique, potentially leading to over-interpretation of results of the relations between constructs. Future studies could investigate the same variables in a longitudinal design in order to better explore the cause and effect relations among the study variables and include data from multiple informants in order to decrease shared method variance and to have more information about the parent-child relationships being studied. The current study did not control for the other parents' support in each model, making it impossible to know how the support from each parent influences the relations between parental support and other variables in each model and every day within the family context. Future studies should consider controlling for the other parents' support or including variables that represent both parents within the same models.

In addition, the use of latent profile analysis comes with a few limitations that must be mentioned. First, identifying the profiles may be subject to selection bias on the part of the researcher as there is not yet a set standard in the literature for making decisions about profile indices and acceptable group size. Second, the profiles that were identified in this sample may not be generalizable to a wider population; rather, they may be profiles specific to this sample of U.S. Latino/a college students that cannot be generalized to the wider U.S. Latino/a young adult population or that may not be able to be replicated in future studies with different samples.

Another set of limitations is concerning the sample. All participants were enrolled in a four-year college, which only allows the results to be generalized to U.S. Latino/a 
college students, and this sample does not capture the experiences of the majority of U.S. Latino/a young adults, who are not attending four-year colleges. According to the Pew Research Center (Krogstad, 2016), as of 2014, 35\% of Hispanic young adults between the ages of 18 and 24 were enrolled in either a two or four-year college. This percentage had risen from 22\% in 1993 (Krogstad, 2016), which indicates that more and more U.S. Latino/as are attending college. However, in general, U.S. Latino/a college students tend to be a generally well-functioning subgroup of U.S. Latino/a young adults, so what about the other $65 \%$ of U.S. Latino/a young adults, who may be facing contextual or economic barriers to higher education? Research by the Pew Research Center (Krogstad, 2016) discusses the fact that many U.S. Latino/a young adults in this age range cite financial reasons for not attending college, so they may choose other paths, such as getting a job or joining the military, to help provide for themselves and their family. If researchers in academia continue to often rely on convenience samples of college students, even if those samples are gathered purposively to be more representative, the participants were still able to attain attending college and do not necessarily represent the experiences of U.S. Latino/a young adults.

Although the current sample does a better job of representing more Latino/a subgroups within the United States, the representation from some of the subgroups, such as Central American (5.6\%) and Cuban/Cuban American (3.6\%), was small, and others were not represented at all, such as Haitian/Haitian American. For example, the subgroup indicated as Central American would still be comprised of participants from a variety of Central American countries that have different histories and immigration experiences. When thinking about the groups of the largest origin in the United States, not including 
Mexican and Puerto Rican origin, five other subgroups have populations greater than 1 million: Salvadorans, Cubans, Dominicans, Guatemalans, and Columbians (Flores, 2017). However, in this data collection project, Salvadoran, Guatemalan, and Columbianorigin participants would all be grouped into broader categories.

Approximately half of the participants reported being of Mexican origin, so results drawn from this data still are strongly representative of the experiences of U.S. Latino/as of Mexican origin. Yet U.S. Latino/a subgroups face both health and academic disparities, so it is important to study each subgroup's experiences separately. For example, a study by Zsembik and Fennell (2005) found variations in health outcomes among U.S. Latino/a subgroups such that U.S. Mexicans tended to fare better, Puerto Rican Americans tended to fare worse, and U.S. Cubans and U.S. Dominicans had mixed health outcomes. The reasons why and the ways in which U.S. Latino/a families immigrated to the United States may play a role in this, driven by disparities in socioeconomic status and education both before leaving the country of origin and after immigrating to the United States. For example, Fuligni and Perreira (2009) report that immigrants from Central America and Mexico tend to have the lowest levels of both education and income out of the U.S. Latino/a subgroups, whereas immigrants from South America are more likely to have been professionals in their home country who immigrated via employment visas; thus, U.S. South Americans may have greater opportunities based on family educational background and socioeconomic status (Fuligni \& Perreira, 2009). Thus, these critiques suggest the need to investigate each U.S. Latino/a subgroup independently to better understand the experiences shaping the lives of participants from each subgroup. 
Lastly, the sample size also limited the ability to do multigroup latent profile analysis, which could have revealed interesting differences by gender, considering gender was significantly correlated with empathic concern. Future studies could make an attempt to sample approximately equal numbers of men and women in order to be able to further investigate the role of gender in these relations.

\section{Conclusions and Future Directions}

Overall, this study provides some important additions to the literature on prosocial behaviors, such as proposing a new heuristic model for better understanding the interplay of individual, relational, and cultural-level correlates of prosocial behaviors. The results of this study support the continued use of variable-centered models in order to better understand how pairs of variables interact with one another. For example, the variablecentered analyses supported the important role of parental support from both mothers and fathers in promoting U.S. Latino/a young adults' socioemotive and sociocognitive traits, as well as the cultural value of familism. Yet it appears that the roles of maternal and paternal support manifest in slightly different ways that require further investigation. Future research and theory development should continue to include a variable-centered approach in order to understand how each correlate of prosocial behaviors directly and indirectly affects prosocial behaviors. Because of the differences identified in maternal and parental support models, future theory and research should include multiple socialization influences, both within the family and beyond, to gather a more complete picture of the correlates of unique prosocial behaviors. In addition, these differences can be used to inform intervention studies with U.S. Latino/a college students in the family context, such as intervention programs that look at the role of mothers, fathers, teachers, 
or friends in promoting socioemotive and sociocognitive traits, and ultimately, prosocial behaviors.

This study also supports the use of latent profile analysis to help understand how the interplay of variables at each level of influence may look different for various groups of people in the real world. Not everyone's lived experiences are going to look the same, and distinct combinations of levels of these variables could lead to different results for various groups of the population. Thus, in order to better capture how variables may relate differently for unique groups of people, latent profile analysis provides important additional information to grow the field beyond an understanding of how variables relate overall. Future theory and research on prosocial behaviors can build on these ideas by studying how multiple variables interrelate to affect both types and targets of prosocial behaviors. The identification of different groups could also help inform interventions by suggesting how even if participants score low on a certain variable, by supporting their growth on other variables, prosocial behaviors could still be promoted by other means. For example, intervention programs for U.S. Latino/a college students who report low levels of parental support could focus on bolstering their sociocognitive and socioemotive traits, as well as cultural values, by other socialization means, such as through mentorship programs.

The results of this study and the experience gained by conducting this project provide an increased motivation to further explore the use of the proposed heuristic model to better understand the levels of influence that simultaneously affect prosocial behaviors. New data collection projects that address the limitations discussed above, such as measuring more relationship qualities, more relationships, and more specific details 
about the targets of prosocial behaviors would likely be fruitful in continuing to fill gaps in the literature. Future studies should also consider controlling for the other parents' support in each model (i.e., control for paternal support in the maternal support models and control for maternal support in the paternal support models) in order to control for the potential influence of the other parent on relations between parenting variables and any other variables. In addition, longitudinal studies could be used to see how relationship qualities and dynamics change over time, as well as how macro-level contextual factors change over time. This heuristic model is purposefully broad so that it can be used as a guide for future studies that interchange variables at all three levels in order to keep contributing to an understanding of how all correlates of prosocial behaviors coexist within individuals at the same time. In the real world, in the moment that a person makes a decision about whether or not to help, it is impossible to extract just their empathic concern, their perspective taking, or how their support from that person affected their decision. Instead, continuing to add to the literature that captures the simultaneous effects of multiple variables can help move the field forward towards a more real-world understanding of prosocial behaviors. By doing so, research on prosocial behaviors can be more useful to practitioners, educators, parents, and others in order to make informed decisions about where to focus interventions and social programs. By doing so, researchers can hopefully use the literature on prosocial behaviors to give back to the people who have participated in research studies over the years and to the future generations that come after them — by helping make real people's lives and lived experiences better. 


\section{References}

Bandura, A. (1986). Social foundations of thought and action: A social cognitive theory. Englewood Cliffs, NJ: Prentice-Hall.

Batson, C. D. (1998). Altruism and prosocial behavior. In D. T. Gilbert, S. T. Fiske, \& G. Lindzey (Eds.), The handbook of social psychology (4th ed.) (pp. 282-316). Boston, MA: McGraw-Hill.

Bronfenbrenner, U. (1995). Developmental ecology through space and time: A future perspective. In P. Moen, G. H. Elder, Jr., \& K. Lüscher (Eds.), Examining lives in context: Perspectives on the ecology of human development (pp. 619-647).

Washington, DC, US: American Psychological Association.

Bronfenbrenner, U. (1999). Environments in developmental perspective: Theoretical and operational models. In S. L. Friedman \& T. D. Wachs (Eds.), Measuring environment across the life span: Emerging methods and concepts (pp. 3-28). Washington, DC, US: American Psychological Association.

Bronstein, P. (1994). Patterns of parent-child interaction in Mexican families: A crosscultural perspective. International Journal of Behavioral Development, 17(3), 423-446.

Browne, M. W., \& Cudeck, R. (1993). Alternative ways of assessing model fit. In K. A. Bollen \& J. S. Long (Eds.), Testing structural equation models (pp. 136-162). Newbury Park, CA: Sage.

Bugental, D. B., \& Goodnow, J. G. (1998). Socialization processes. In W. Damon \& R. M. Lerner (Series Eds.), \& N. Eisenberg (Vol. Ed.), Handbook of child psychology: Vol. 3 Social, emotional, and personality development (6th ed., pp. 
366-428). Hoboken, NJ: Wiley.

Cabrera, N. J., \& Bradley, R. H. (2012). Latino fathers and their children. Child Development Perspectives, 6(3), 232-238.

Calderón-Tena, C. O., Knight, G. P., \& Carlo, G. (2011). The socialization of prosocial behavioral tendencies among U.S. Mexican adolescents: The role of familism values. Cultural Diversity and Ethnic Minority Psychology, 17(1), 98-106.

Carlo, G., \& de Guzman, M. R. T. (2009). Theories and research on prosocial competencies among US Latinos/as. In F. A. Villarruel, G. Carlo, J. M. Grau, M. Azmitia, N. J. Cabrera, \& T. J. Chahin (Eds.), Handbook of US Latino psychology: Developmental and community-based perspectives (pp. 191-211). Thousand Oaks, CA: Sage Publications, Inc.

Carlo, G., Hausmann, A., Christiansen, S., \& Randall, B. A. (2003). Sociocognitive and behavioral correlates of a measure of prosocial tendencies for adolescents. Journal of Early Adolescence, 23(1), 107-134.

Carlo, G., Knight, G. P., Basilio, C. D., \& Davis, A. N. (2014). Predicting prosocial tendencies among U.S. Mexican youth. In L. M. Padilla-Walker \& G. Carlo (Eds.), Prosocial development: A multidimensional approach (pp. 242-257). New York, NY: Oxford University Press.

Carlo, G., Knight, G. P., McGinley, M., \& Hayes, R. (2011). The roles of parental inductions, moral emotions, and moral cognitions in prosocial tendencies among Mexican American and European American early adolescents. Journal of Early Adolescence, 31(6), 757-781.

Carlo, G., Knight, G. P., McGinley, M., Hayes, R., \& Shen, Y. L. (2009). The role of 
values in prosocial moral development: Validation of a measure of prosocial values to use with U.S. Mexican early adolescents. In M. C. Richaud \& J. E. Moreno (Eds.), Investigación en Ciencias del Comportamiento: Avances Iberoamericanos, Tomo II (Investigations in the Behavioral Sciences: IberoAmerican Advances, Vol. 2) (pp. 1029-1050). Buenos Aires: Ediciones CIIPMECONICET.

Carlo, G., Knight, G. P., McGinley, M., Zamboanga, B. L., \& Jarvis, L. H. (2010). The multidimensionality of prosocial behaviors and evidence of measurement equivalence in Mexican American and European American early adolescents. Journal of Research on Adolescence, 20(2), 334-358.

Carlo, G., McGinley, M., Hayes, R. C., \& Martinez, M. M. (2012). Empathy as a mediator of the relations between parent and peer attachment and prosocial and physically aggressive behaviors in Mexican American college students. Journal of Social and Personal Relationships, 29(3), 337-357.

Carlo, G., \& Pierotti, S. L. (2020). The development of prosocial motives. In L. A. Jensen (Ed.), Oxford handbook of moral development: An interdisciplinary perspective. Oxford University Press.

Carlo, G., \& Randall, B. A. (2002). The development of a measure of prosocial behaviors for late adolescents. Journal of Youth and Adolescence, 31(1), 31-44.

Carlo, G., White, R. M., Streit, C., Knight, G. P., \& Zeiders, K. H. (2018). Longitudinal relations among parenting styles, prosocial behaviors, and academic outcomes in US Mexican adolescents. Child Development, 89(2), 577-592.

Davis, M. H. (1983). Measuring individual differences in empathy: Evidence for a 
multidimensional approach. Journal of Personality and Social Psychology, 44(1), 113-126.

Davis, A. N., Carlo, G., \& Knight, G. P. (2015). Perceived maternal parenting styles, cultural values, and prosocial tendencies among Mexican American youth. The Journal of Genetic Psychology, 176(4), 235-252.

Davis, A. N., Carlo, G., Streit, C., \& Crockett, L. J. (2018). Considering economic stress and empathic traits in predicting prosocial behaviors among U.S. Latino adolescents. Social Development, 27(1), 58-72.

de Guzman, M. R. T., Carlo, G., \& Edwards, C. P. (2008). Prosocial behaviors in context: Examining the role of children's social companions. International Journal of Behavioral Development, 32(6), 522-530.

de Guzman, M. R. T., Edwards, C. P., \& Carlo, G. (2005). Prosocial behaviors in context: A study of the Gikuyu children of Ngecha, Kenya. Journal of Applied Developmental Psychology, 26, 542-558.

Eisenberg, N. (1991). Meta-analytic contributions to the literature on prosocial behavior. Personality and Social Psychology Bulletin, 17, 273-282.

Eisenberg, N., Fabes, R. A., \& Spinrad, T. L. (2006). Prosocial behavior. In W. Damon \& R. M. Lerner (Series Eds.) \& N. Eisenberg (Vol. Ed.), Handbook of child psychology: Vol. 3, Social, emotional, and personality development (6th ed., pp. 646-718). New York: Wiley.

Feshbach, N. D. (1987). Parental empathy and child adjustment/maladjustment. In N. Eisenberg \& J. Strayer (Eds.), Empathy and its development (pp. 271-291). New York: Cambridge University Press. 
Flores, A. (2017). How the U.S. Hispanic population is changing. Washington, DC: Pew Research Center. http://www.pewresearch.org/fact-tank/2017/09/18/how-the-u-shispanic-population-is-changing/

Fuligni, A. J., \& Perreira, K. M. (2009). Immigration and adaptation. In F. A. Villarruel, G. Carlo, J. M. Grau, M. Azmitia, N. J. Cabrera, \& T. J. Chahin (Eds.), Handbook of U.S. Latino psychology: Developmental and community-based perspectives (pp. 99-113). Thousand Oaks, CA: Sage.

Furman, W., \& Buhrmester, D. (1985). Children's perceptions of the personal relationships in their social networks. Developmental Psychology, 21(6), 10161024.

García Coll, C., Crnic, K., Lamberty, G., Wasik, B. H., Jenkins, R., Garcia, H. V., \& McAdoo, H. P. (1996). An integrative model for the study of developmental competencies in minority children. Child Development, 67(5), 1891-1914.

George, D., \& Mallery, P. 2003. SPSS for Windows step by step: a simple guide and reference, Boston, MA: Allyn and Bacon.

Grusec, J. E., Chaparro, M. P., Johnston, M., \& Sherman, A. (2014). The development of moral behavior from a socialization perspective. In M. Killen \& J. G. Smetana (Eds.), Handbook of Moral Development (pp. 113-134). United States: Psychology Press.

Grusec, J. E., \& Goodnow, J. J. (1994). Impact of parental discipline methods on the child's internalization of values: A reconceptualization of current points of view. Developmental Psychology, 30, 4-19.

Guevara, I. P., Cabrera, V. E., Gonzalez, M. R., \& Devis, J. V. (2015). Empathy and 
sympathy as mediators between parental inductive discipline and prosocial behavior in Colombian families. International Journal of Psychological Research, $8(2), 34-48$.

Hoffman, M. L. (2001). Empathy and moral development: Implications for caring and justice. Cambridge, UK: Cambridge University Press.

Hofstede, G. (1984). Culture's consequences: International differences in work-related values (Abridged ed.). Beverly Hills, CA: Sage.

IBM Corp. Released 2016. IBM SPSS Statistics for Windows, Version 24.0. Armonk, NY: IBM Corp.

Kline, R. B. (1998). Principles and practice of structural equation modeling (1st ed.) New York, NY: The Guilford Press.

Kline, R. B. (2011). Principles and practice of structural equation modeling (3rd ed.). New York, NY: The Guilford Press.

Knight, G. P., Berkel, C., Carlo, G., \& Basilio, C. (2011). The socialization of culturally related values and the mental health outcomes of Latino youth. In N. Cabrera, F. A. Villarruel, \& H. E. Fitzgerald (Eds.), Latina and Latino children's mental health: Vol. 2, Prevention and treatment (pp. 109-131). Santa Barbara, CA: Praeger.

Knight, G. P., Bernal, M. E., \& Carlo, G. (1995). Socialization and the development of cooperative, competitive, and individualistic behaviors among Mexican American children. In E. E. García \& B. McLaughlin (Eds.), Meeting the challenge of linguistic and cultural diversity in early childhood education (Vol. 6, pp. 85-102). New York, NY: Teachers College Press. 
Knight, G. P., Carlo, G., Basilio, C. D., \& Jacobson, R. P. (2014). Familism values, perspective taking, and prosocial moral reasoning: Predicting prosocial tendencies among Mexican American adolescents. Journal of Research on Adolescence, 25(4), 717-727.

Knight, G. P., Carlo, G., Mahrer, N. E., \& Davis, A. N. (2016). The socialization of culturally related values and prosocial tendencies among Mexican-American adolescents. Child Development, 87(6), 1758-1771.

Knight, G. P., Gonzales, N. A., Saenz, D. S., Bonds, D. D., Germán, M., Deardorff, J., ... \& Updegraff, K. A. (2010). The Mexican American cultural values scale for adolescents and adults. The Journal of Early Adolescence, 30(3), 444-481.

Knight, G. P., \& Kagan, S. (1977). Development of prosocial and competitive behaviors in Anglo-American and Mexican-American children. Child Development, 48, 1385-1394.

Kohlberg, L. (1976). Moral stages and moralization: The cognitive-development approach. Moral development and behavior: Theory research and social issues, 31-53.

Krogstad, J. M. (2016) 5 facts about Latinos and education. Washington, DC: Pew Research Center. https://www.pewresearch.org/fact-tank/2016/07/28/5-factsabout-latinos-and-education/

Markus, H. R., \& Hamedani, M. G. (2007). Sociocultural psychology: The dynamic interdependence among self systems and social systems. In S. Kitayama \& D. Cohen (Eds.), Handbook of cultural psychology (pp. 3-39). New York, NY: Guilford Press. 
Matsumoto, D. (1996). Culture and psychology. Pacific Grove, CA: Brooks/Cole Publishing Company.

McLoyd, V. C., Cauce, A. M., Takeuchi, D., \& Wilson, L. (2000). Marital processes and parental socialization in families of color: A decade review of research. Journal of Marriage and Family, 62(4), 1070-1093.

Muthén, L. K., \& Muthén, B. O. (2010). Mplus user's guide (6th ed.). Los Angeles, CA. Padilla-Walker, L. M., \& Carlo, G. (2014). Prosocial behavior: Past, present, and future. In L. M. Padilla-Walker \& G. Carlo (Eds.), Prosocial behavior: A multidimensional approach (pp. 3-16). New York, NY: Oxford University Press.

Pierotti, S. L., Carlo, G., \& Conejo, L. D. (2017, July). Does active coping play a role in prosocial behavior? A study of Costa Rican and United States college students' socioemotional and sociocognitive correlates of prosocial behavior. Poster presented at the International Society for the Study of Behavioural Development Regional Workshop on Positive Youth Development in Times of Social Change, Syros, Greece.

Raffaelli, M., Carlo, G., Carranza, M. A., \& Gonzalez-Kruger, G. E. (2005). Understanding Latino children and adolescents in the mainstream: Placing culture at the center of developmental models. New Directions for Child and Adolescent Development, 2005(109), 23-32.

Schwartz, S. H. (1992). Universals in the content and structure of values: Theoretical advances and empirical tests in 20 countries. In M. P. Zanna (Ed.), Advances in experimental social psychology (Vol. 25, pp. 1-65). San Diego, CA: Academic Press. 
Schwartz, S. H., \& Bilsky, W. (1987). Toward a universal psychological structure of human values. Journal of Personality and Social Psychology, 53, 550-562.

Shen, Y. L., Carlo, G., \& Knight, G. P. (2013). Relations between parental discipline, empathy-related traits, and prosocial moral reasoning: A multicultural examination. The Journal of Early Adolescence, 33(7), 994-1021.

Stanley, L., Kellermanns, F. W., \& Zellweger, T. M. (2017). Latent Profile Analysis: Understanding family firm profiles. Family Business Review, 30(1), 84-102.

Stein, G. L., Gonzalez, L. M., \& Huq, N. (2012). Cultural stressors and the hopelessness model of depressive symptoms in Latino adolescents. Journal of Youth and Adolescence, 41(10), 1339-1349.

Super, C. M., \& Harkness, S. (1986). The developmental niche: A conceptualization at the interface of child and culture. International Journal of Behavioral Development, 9, 545-569.

Tein, J. Y., Coxe, S., \& Cham, H. (2013). Statistical power to detect the correct number of classes in latent profile analysis. Structural Equation Modeling: A Multidisciplinary Journal, 20(4), 640-657.

Whiting, B. B. (1980). Culture and social behavior: A model for the development of social behavior. Ethos, 8(2), 95-116.

Whiting, B. B., \& Whiting, J. W. M. (1975). Children of six cultures: A psycho-cultural analysis. Cambridge, MA: Harvard University Press.

U.S. Census Bureau (2017). Facts for features: Hispanic Heritage Month 2017. Washington, DC: United States Census Bureau. https://www.census.gov/ newsroom/facts-for-features/2017/hispanic-heritage.html 
Zsembik, B. A., \& Fennell, D. (2005). Ethnic variation in health and the determinants of health among Latinos. Social Science \& Medicine, 61(1), 53-63. 
Appendix A

Heuristic and Conceptual Models

\section{Figure 1}

\section{Heuristic Model}

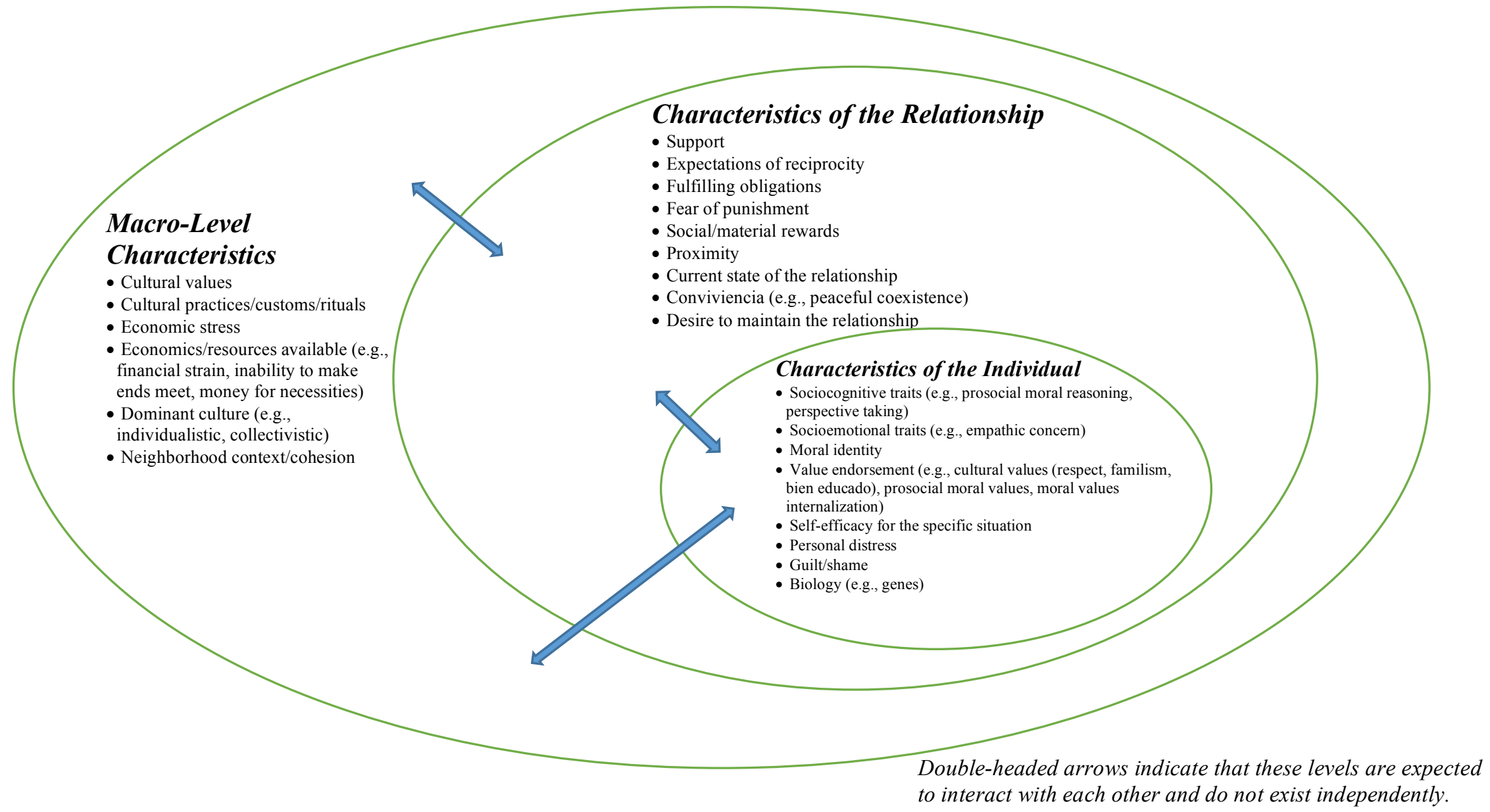




\section{Figure 2}

Conceptual Model for the Present Study

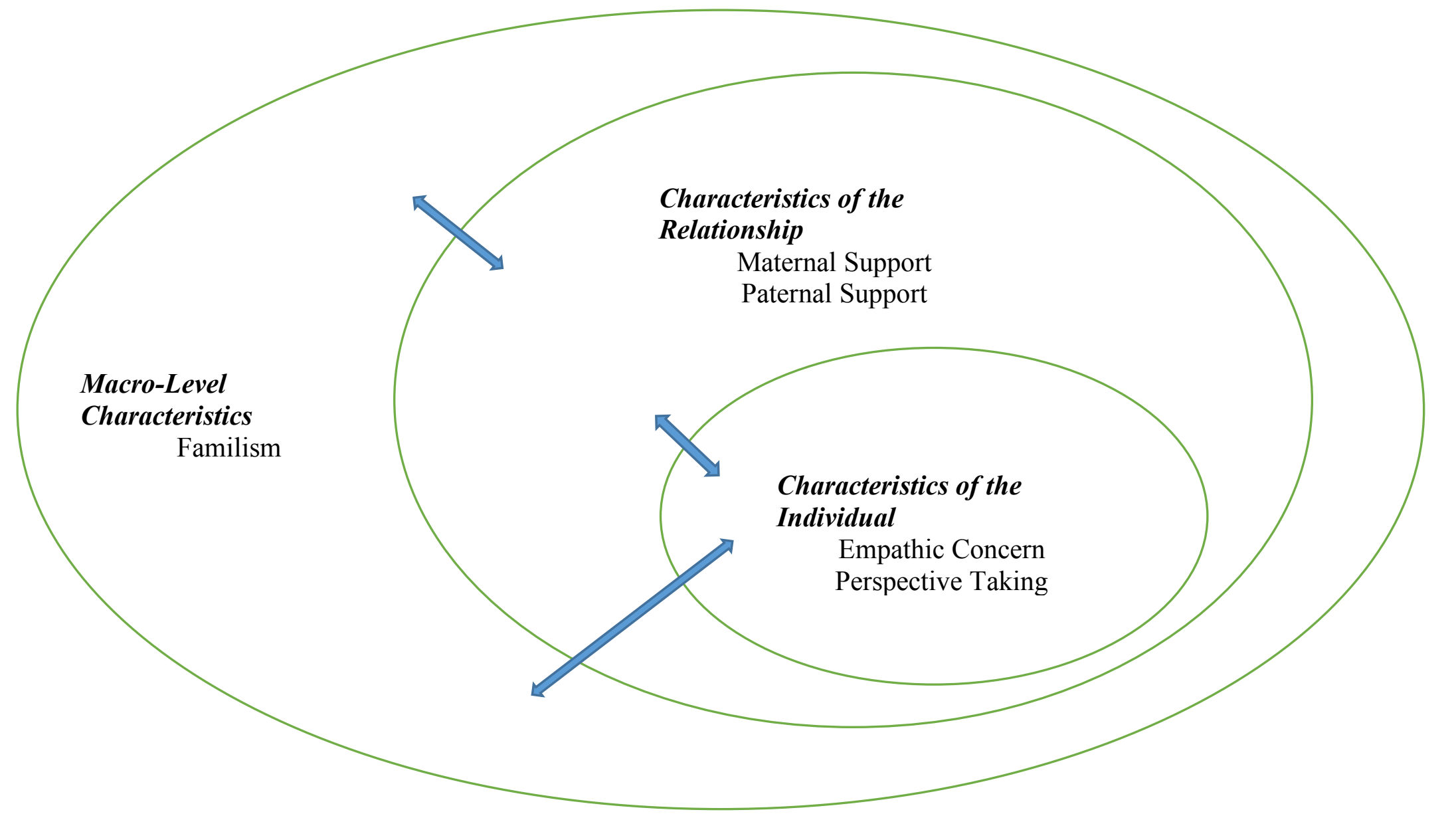

Double-headed arrows indicate that these levels are expected to interact with each other and do not exist independently. 


\section{Appendix B}

Descriptive Statistics and Bivariate Correlations

\section{Table 1}

Correlations and Descriptive Statistics for Study Variables $(N=250)$

\begin{tabular}{|c|c|c|c|c|c|c|c|c|c|c|c|c|c|}
\hline & 1 & 2 & 3 & 4 & 5 & 6 & 7 & 8 & 9 & 10 & 11 & 12 & 13 \\
\hline 1. Familism & - & & & & & & & & & & & & \\
\hline 2. Empathic Concern & $.17 *$ & - & & & & & & & & & & & \\
\hline 3. Perspective Taking & .11 & $.61 * * *$ & - & & & & & & & & & & \\
\hline 4. Maternal Support & $.39 * * *$ & $.20 * *$ & $.24 * * *$ & - & & & & & & & & & \\
\hline 5. Paternal Support & $.36 * * *$ & $.14^{*}$ & $.14^{*}$ & $.68 * * *$ & - & & & & & & & & \\
\hline $\begin{array}{ll}\text { 6. } & \text { Dire Prosocial } \\
\text { Behaviors }\end{array}$ & $.32 * * *$ & $.54 * * *$ & $.50 * * *$ & $.31 * * *$ & $.20 * *$ & - & & & & & & & \\
\hline $\begin{array}{l}\text { 7. } \\
\text { Prosocional } \\
\text { Behaviors }\end{array}$ & $.28 * * *$ & $.57 * * *$ & $.47 * * *$ & $.31 * * *$ & $.20 * *$ & $.80 * * *$ & - & & & & & & \\
\hline $\begin{array}{l}\text { 8. Compliant } \\
\text { Prosocial } \\
\text { Behaviors }\end{array}$ & $.37 * * *$ & $.53 * * *$ & $.43 * * *$ & $.37 * * *$ & $.25 * * *$ & $.80 * * *$ & $.75 * * *$ & - & & & & & \\
\hline $\begin{array}{ll}\text { 9. } & \text { Prosocial } \\
\text { Behaviors towards } \\
\text { Family }\end{array}$ & $.38 * * *$ & $.47 * * *$ & $.42 * * *$ & $.34 * * *$ & $.22 * *$ & $.85 * * *$ & $.80 * * *$ & $.79 * * *$ & - & & & & \\
\hline $\begin{array}{l}\text { 10. Prosocial } \\
\text { Behaviors towards } \\
\text { Friends }\end{array}$ & $.30 * * *$ & $.49 * * *$ & $.40 * * *$ & $.26 * * *$ & .11 & $.82 * * *$ & $.83 * * *$ & $.80 * * *$ & $.79 * * *$ & - & & & \\
\hline $\begin{array}{l}\text { 11. Prosocial } \\
\text { Behaviors towards } \\
\text { Strangers }\end{array}$ & $.22 * *$ & $.55 * * *$ & $.47 * * *$ & $.29 * * *$ & $.27 * * *$ & $.77 * * *$ & $.82 * * *$ & $.72 * * *$ & $.54 * * *$ & $.56^{* * *}$ & - & & \\
\hline 12. Gender & -.04 & $.25 * * *$ & .05 & .12 & -.02 & .09 & $.19 * *$ & $.17 * *$ & $.17 * *$ & $.14^{*}$ & .11 & - & \\
\hline 13. Nativity & -.03 & -.03 & -.01 & .04 & .11 & -.08 & -.04 & -.03 & -.03 & -.06 & -.05 & .10 & - \\
\hline Mean & 3.68 & 4.56 & 4.44 & 3.64 & 3.29 & 3.67 & 3.40 & 3.60 & 3.75 & 3.87 & 2.97 & - & - \\
\hline$S D$ & .77 & 1.04 & .89 & .94 & 1.00 & .77 & .80 & .79 & .78 & .76 & 1.01 & - & - \\
\hline
\end{tabular}




\section{Appendix C}

\section{Variable-Centered Models}

\section{Figure 3}

Intervening Mechanisms in the Relations between Maternal Support and Three Types of Prosocial Behaviors $(N=247)$

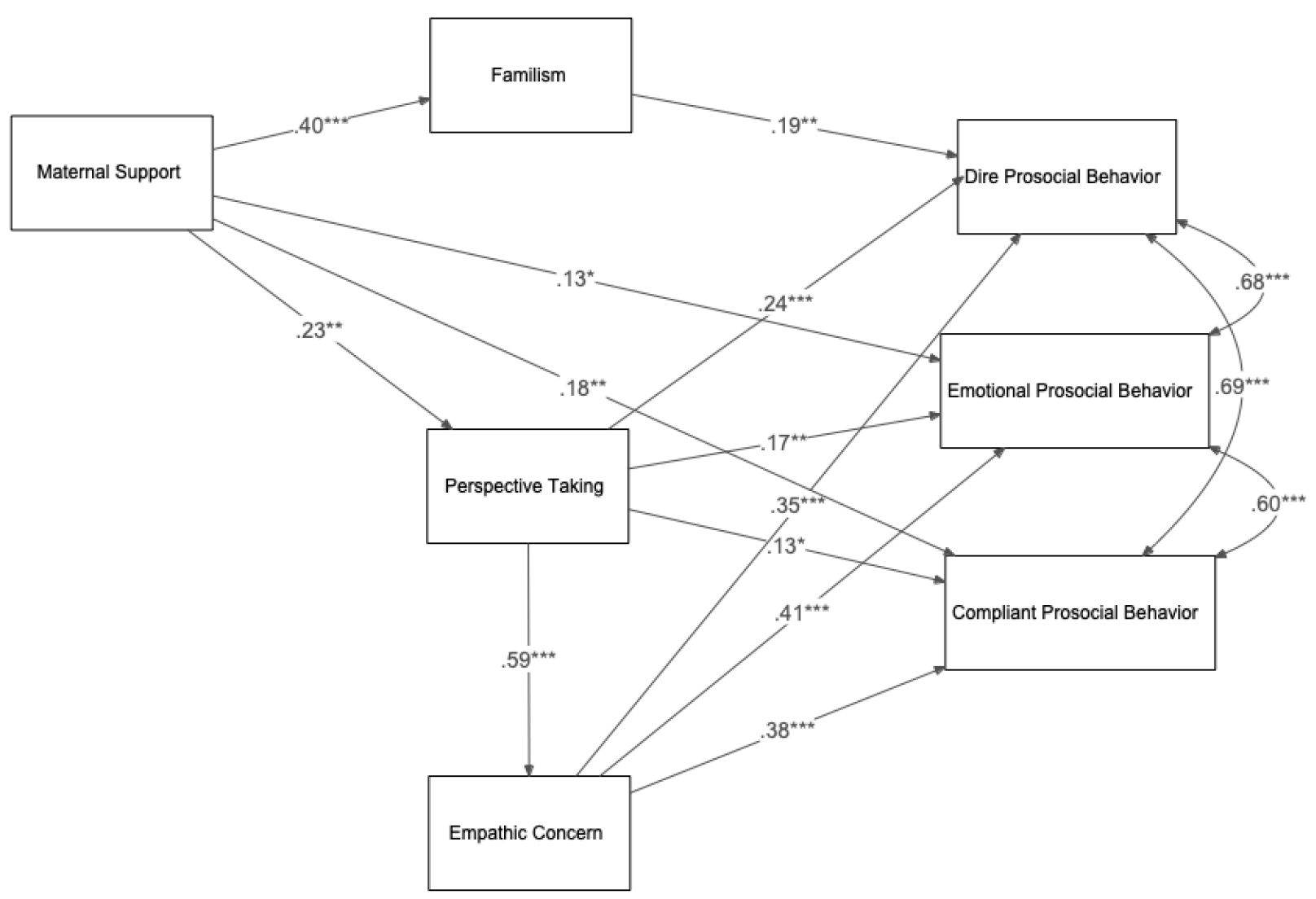

Note: $* p<.05 ; * * p<.01 ; * * * p<.001$ 


\section{Figure 4}

Intervening Mechanisms in the Relations between Paternal Support and Three Types of

Prosocial Behaviors $(N=245)$

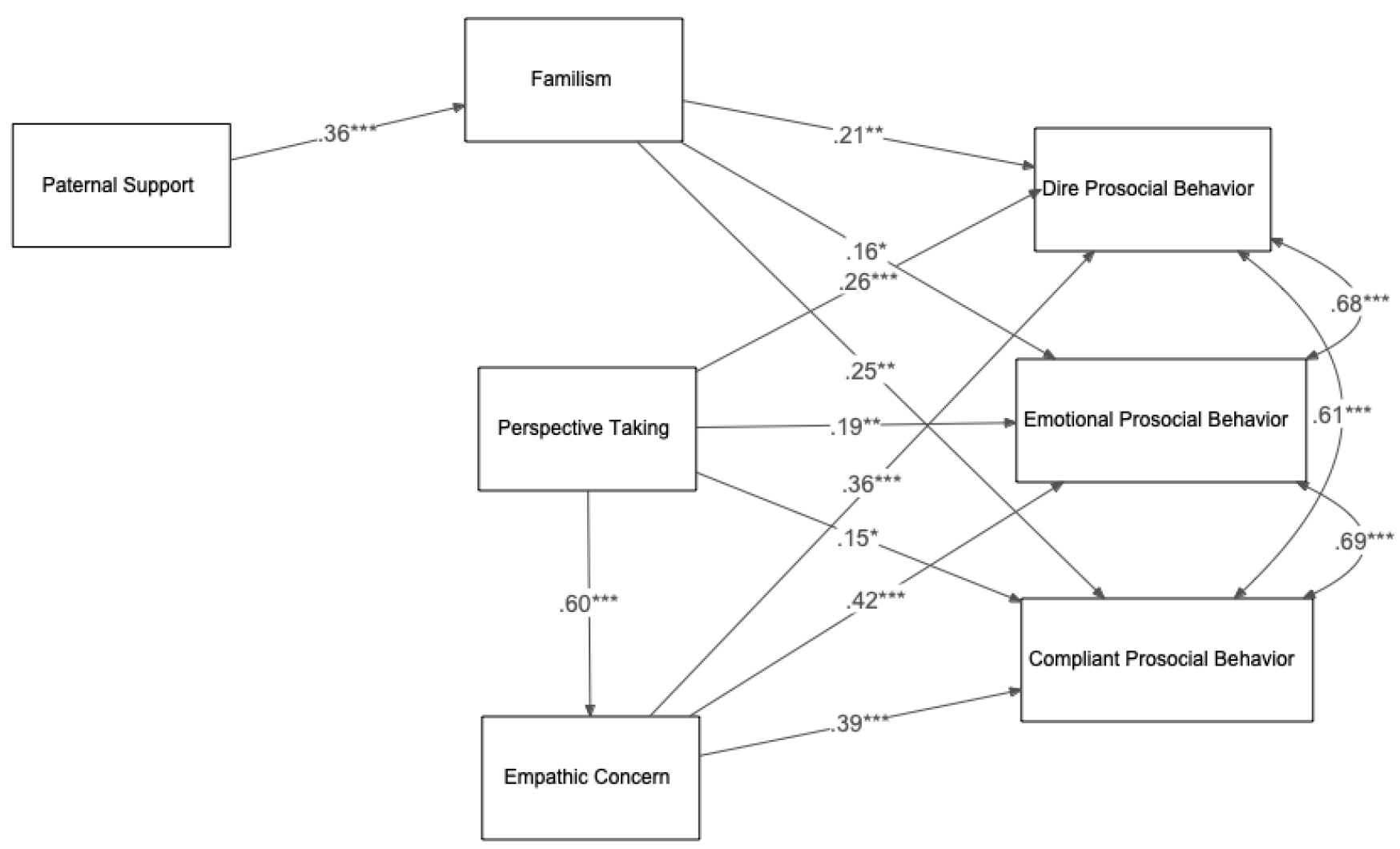

Note: ${ }^{*} p<.05 ;{ }^{* *} p<.01 ;{ }^{* * *} p<.001$ 


\section{Figure 5}

Intervening Mechanisms in the Relations between Maternal Support and Three Unique Targets of Prosocial Behaviors $(N=247)$

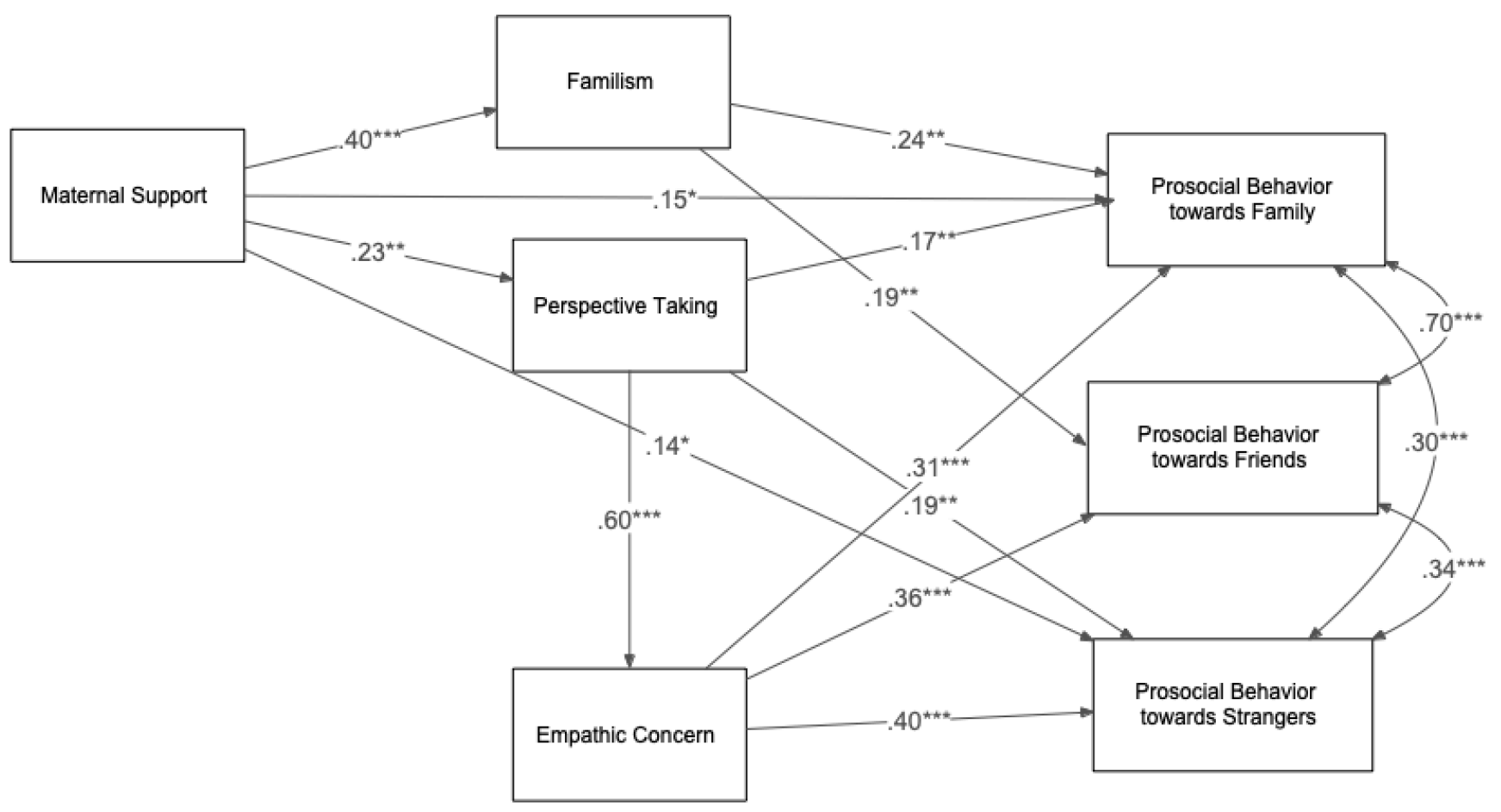

Note: ${ }^{*} p<.05 ; * * p<.01 ; * * * p<.001$ 


\section{Figure 6}

Intervening Mechanisms in the Relations between Paternal Support and Three Unique Targets of Prosocial Behaviors $(N=245)$

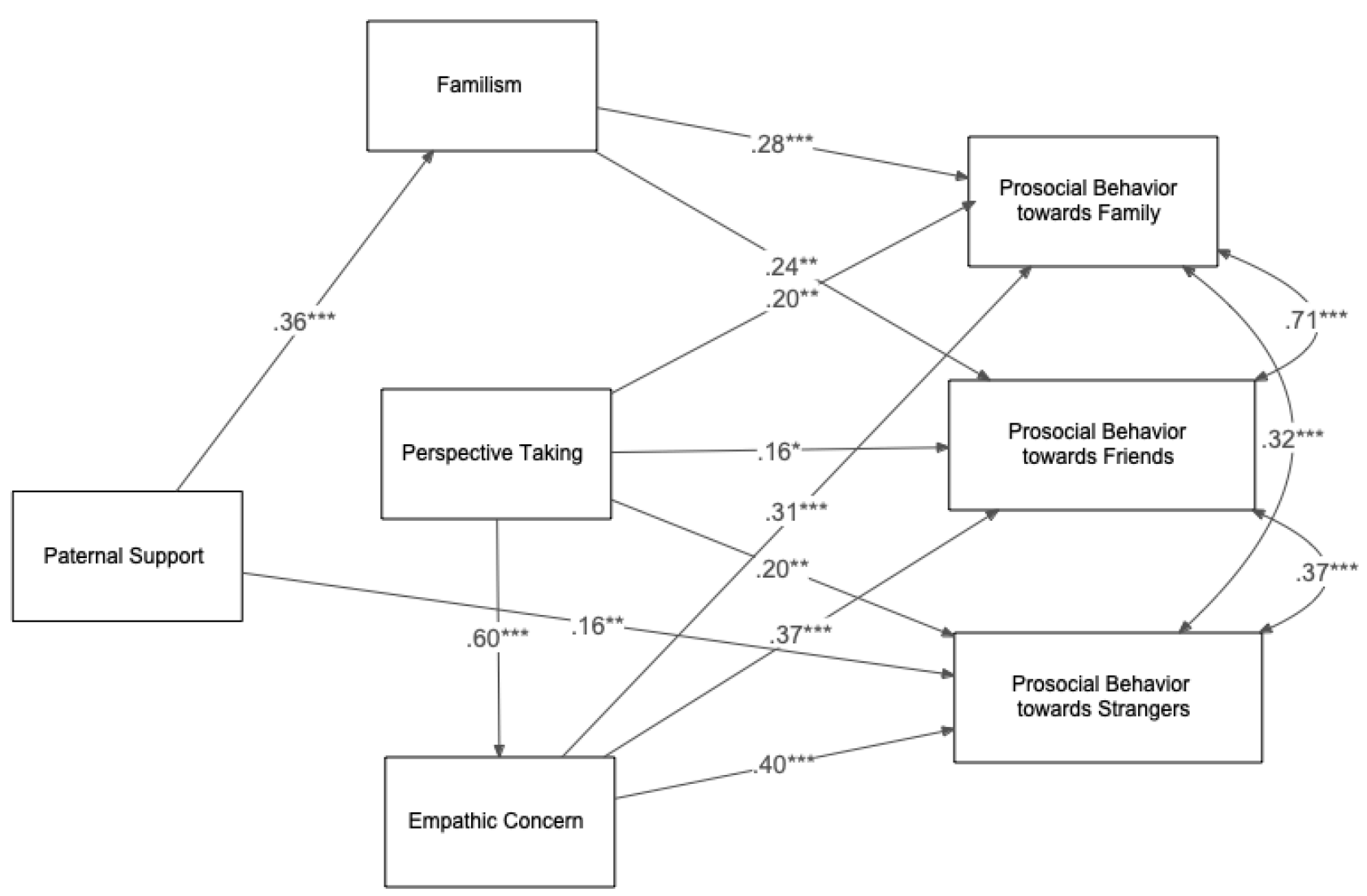

Note: ${ }^{*} p<.05 ;{ }^{* *} p<.01 ; * * * p<.001$ 


\section{Appendix D}

Person-Centered Models

Figure 7

Latent Profile Indicators

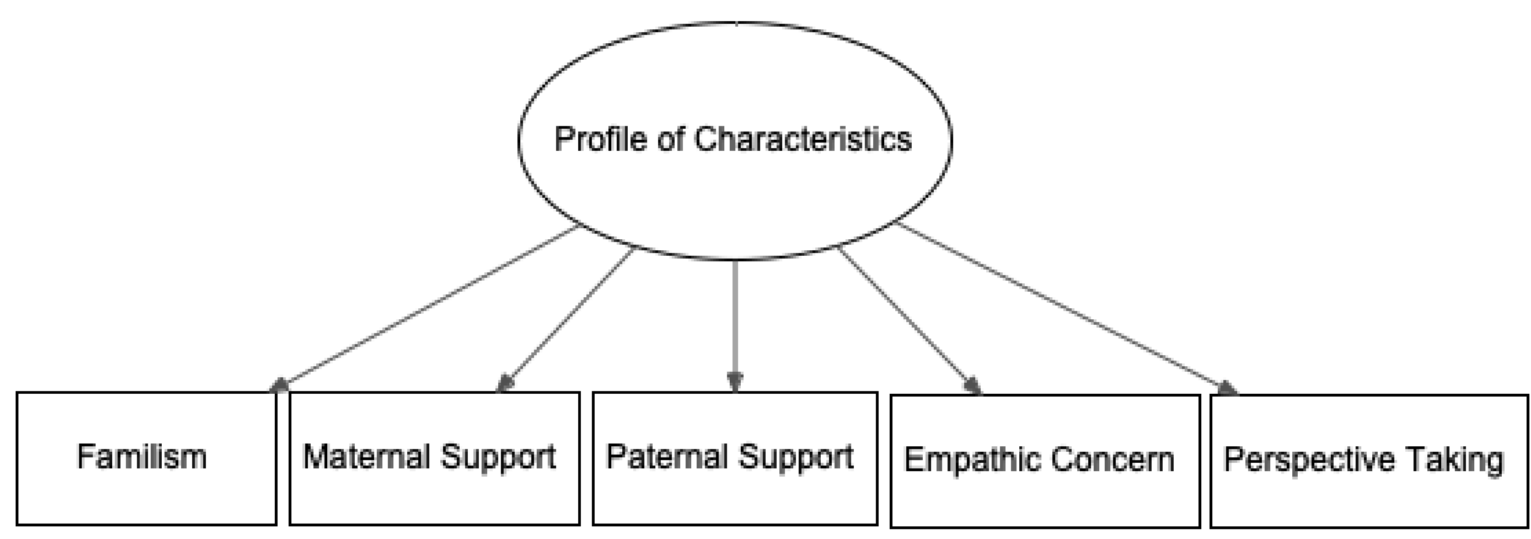


Table 2

Latent Profile Analysis with Only Maternal Support Representing Relational Characteristics

\begin{tabular}{|l|l|l|l|l|l|}
\hline Profiles & BIC & ABIC & Entropy & $\begin{array}{l}\text { LMRT } p- \\
\text { value }\end{array}$ & $\begin{array}{l}\text { Profile } \\
\text { Probabilities }\end{array}$ \\
\hline 1 & 2649.88 & 2624.52 & \multicolumn{1}{|c|}{-} & \multicolumn{1}{c|}{-} & - \\
\hline 2 & 2548.90 & 2507.69 & .73 & $p<.01$ & $.88, .94$ \\
\hline 3 & 2525.96 & 2468.90 & .80 & $p=\mathrm{ns}$ & $.88, .89, .93$ \\
\hline 4 & 2527.39 & 2454.48 & .73 & $p<.05$ & $\begin{array}{l}.81, .88, .78, \\
.91\end{array}$ \\
\hline 5 & & & & & $\begin{array}{l}.81, .93, .87, \\
.84, .88\end{array}$ \\
\hline
\end{tabular}


Table 3

Latent Profile Analysis with Only Paternal Support Representing Relational Characteristics

\begin{tabular}{|l|l|l|l|l|l|}
\hline Profiles & BIC & ABIC & Entropy & $\begin{array}{l}\text { LMRT } p- \\
\text { value }\end{array}$ & $\begin{array}{l}\text { Profile } \\
\text { Probabilities }\end{array}$ \\
\hline 1 & 2673.61 & 2648.25 & \multicolumn{1}{|c|}{-} & - & - \\
\hline 2 & 2583.72 & 2542.51 & .72 & $p<.01$ & $.87, .94$ \\
\hline 3 & 2557.12 & 2500.06 & .80 & $p<.01$ & $.93, .89, .88$ \\
\hline 4 & 2555.84 & 2482.93 & .80 & $p=\mathrm{ns}$ & $\begin{array}{l}.89, .92, .85, \\
.85\end{array}$ \\
\hline 5 & & & & $p=\mathrm{ns}$ & $\begin{array}{l}.80, .91, .94, \\
.86, .78\end{array}$ \\
\hline
\end{tabular}


Table 4

Latent Profile Analysis with Both Maternal and Paternal Support Representing Relational

Characteristics

\begin{tabular}{|l|l|l|l|l|c|}
\hline Profiles & BIC & ABIC & Entropy & $\begin{array}{l}\text { LMRT } \boldsymbol{p} \text { - } \\
\text { value }\end{array}$ & $\begin{array}{l}\text { Profile } \\
\text { Probabilities }\end{array}$ \\
\hline 1 & 3356.27 & 3324.57 & \multicolumn{1}{|c|}{-} & - & - \\
\hline 2 & 3200.47 & 3149.75 & .76 & $p<.001$ & $.92, .94$ \\
\hline 3 & 3160.33 & 3090.59 & .80 & $p=$ ns & $.88, .87, .95$ \\
\hline $\mathbf{4}$ & $\mathbf{3 1 3 1 . 5 3}$ & $\mathbf{3 0 4 2 . 7 7}$ & $\mathbf{. 8 3}$ & $\boldsymbol{p}<. \mathbf{0 5}$ & $\begin{array}{l}\mathbf{. 8 8}, \mathbf{. 8 6}, .96, \\
\mathbf{. 9 5}\end{array}$ \\
\hline 5 & & & & $p=\mathrm{ns}$ & $\begin{array}{l}.87, .91, .87, \\
.95, .87\end{array}$ \\
\hline
\end{tabular}




\section{Table 5}

Differences Across LPA Profiles on LPA Indicators and Correlates (N=250)

\begin{tabular}{|c|c|c|c|c|c|}
\hline & $\begin{array}{c}\text { Moderate } \\
\text { Scorers } \\
(n=55)\end{array}$ & $\begin{array}{c}\text { High } \\
\text { Individual- } \\
\text { Level } \\
\text { Variable } \\
\text { Scorers } \\
(n=65)\end{array}$ & $\begin{array}{c}\text { Low } \\
\text { Parental } \\
\text { Support } \\
\text { Scorers } \\
(n=9)\end{array}$ & $\begin{array}{c}\text { High } \\
\text { Scorers } \\
(n=121)\end{array}$ & F Statistic \\
\hline \multicolumn{6}{|l|}{ LPA Indicators } \\
\hline Familism & $3.42_{3,4}$ & $3.65_{3,4}$ & $1.95_{1,2,4}$ & $3.93_{1,2,3}$ & $F(3,237)=26.37, p<.001$ \\
\hline Empathic Concern & $3.27_{2,3,4}$ & $4.98_{1}$ & $4.57_{1}$ & $4.92_{1}$ & $F(3,246)=64.33, p<.001$ \\
\hline Perspective Taking & $3.48_{2,3,4}$ & $4.82_{1}$ & $4.19_{1}$ & $4.70_{1}$ & $F(3,246)=44.04, p<.001$ \\
\hline Maternal Support & $2.96_{3,4}$ & $3.17_{3,4}$ & $1.41_{1,2,4}$ & $4.34_{1,2,3}$ & $F(3,243)=137.60, p<.001$ \\
\hline Paternal Support & $2.92_{2,3,4}$ & $2.38_{1,3,4}$ & $1.20_{1,2,4}$ & $4.08_{1,2,3}$ & $F(3,241)=177.56, p<.001$ \\
\hline \multicolumn{6}{|c|}{ Correlates of Prosocial Behaviors } \\
\hline \multicolumn{6}{|l|}{ Type } \\
\hline Dire & $3.09_{2,4}$ & $3.79_{1}$ & 3.32 & $3.90_{1}$ & $F(3,246)=18.62, p<.001$ \\
\hline Emotional & $2.83_{2,4}$ & $3.49_{1}$ & $2.96_{4}$ & $3.65_{1,3}$ & $F(3,246)=16.98, p<.001$ \\
\hline Compliant & $3.02_{2,4}$ & $3.67_{1}$ & $3.11_{4}$ & $3.85_{1,3}$ & $F(3,246)=18.27, p<.001$ \\
\hline \multicolumn{6}{|l|}{ Target } \\
\hline Family & $3.24_{2,4}$ & $3.83_{1,3}$ & $3.09_{2,4}$ & $3.98_{1,3}$ & $F(3,246)=16.11, p<.001$ \\
\hline Friends & $3.35_{2,4}$ & $4.01_{1}$ & 3.69 & $4.05_{1}$ & $F(3,244)=13.16, p<.001$ \\
\hline Strangers & $2.26_{2,4}$ & 3.021 & 2.57 & $3.30_{1}$ & $F(3,243)=16.53, p<.001$ \\
\hline
\end{tabular}


Appendix E

Measures

\section{Interpersonal Reactivity Index (IRI) \\ Davis (1983)}

\section{Empathic Concern Subscale (7 items)}

1. I often have tender concerned, feelings for people less fortunate than me.

2. Sometimes I don't feel very sorry for other people when they are having problems. (reverse code)

3. When I see someone being taken advantage of, I feel kind of protective towards them.

4. Other people's misfortunes do not usually disturb me a great deal. (reverse code)

5. When I see someone being treated unfairly, I sometimes do not feel very much pity for them. (reverse code)

6. I am often quite touched by things that I see happen.

7. I would describe myself as a pretty soft-hearted person.

\section{Perspective Taking Subscale (7 items)}

1. I sometimes find it difficult to see things from the "other person's" point of view. (reverse code)

2. I try to look at everybody's side of a disagreement before I make a decision.

3. I sometimes try to understand my friends better by imagining how things look from their perspective.

4. If I'm sure I'm right about something, I don't waste time listening to other people's arguments. (reverse code) 
5. I believe that there are two sides to every question and try to look at them both.

6. When I'm upset at someone, I usually try to "put myself in their shoes" for a while.

7. Before criticizing somebody, I try to imagine how I would feel if I were in their place. 


\section{Mexican American Cultural Values Scale (MACVS)}

Knight et al. (2010)

\section{Family Emotional Support Subscale (6 items)}

1. Parents should teach their children that the family always comes first.

2. Family provides a sense of security because they will always be there for you.

3. It is always important to be united as a family.

4. It is important to have close relationships with aunts/uncles, grandparents, and cousins.

5. Holidays and celebrations are important because the whole family comes together.

6. It is important for family members to show their love and affection to one another.

\section{Family Obligations Subscale (5 items)}

1. Children should be taught that it is their duty to care for their parents when their parents get old.

2. If a relative is having a hard time financially, one should help them out if possible.

3. A person should share their home with relatives if they need a place to stay.

4. Older kids should take care of and be role models for their younger brothers and sisters.

5. Parents should be willing to make great sacrifices to make sure their children have a better life.

\section{Family as Referent Subscale (5 items)}

1. Children should always do things to make their parents happy.

2. When it comes to important decisions, the family should ask for advice from close relatives.

3. Children should be taught to always be good because they represent the family.

4. A person should always think about their family when making important decisions. 
5. It is important to work hard and do one's best because this work reflects on the family. 


\section{Network of Relationships Inventory-Short Form \\ Furman \& Buhrmester (1985)}

Support Subscale (7 items for each subscale; completed for mothers and fathers separately)

1. How much does this person treat you like you're admired and respected?

2. How sure are you that this relationship will last no matter what?

3. How much do you play around and have fun with this person?

4. How much does this person help you figure out or fix things?

5. How much do you share your secrets and private feelings with this person?

6. How much does this person really care about you?

7. How much do you take care of this person? 


\section{Prosocial Tendencies Measure \\ Adapted from the PTM-R by Carlo et al. (2003)}

Prosocial Behaviors (9 items for each target of prosocial behaviors; composed of dire, emotional, and compliant subscales towards family, friends, and strangers)

1. It is most fulfilling to me when I can comfort someone (i.e., family/friends/strangers) who is very distressed.

2. I tend to help people (i.e., family/friends/strangers) who are in a real crisis or need.

3. When people (i.e., family/friends/strangers) ask me to help them, I do not hesitate.

4. I tend to help people (i.e., family/friends/strangers) who are hurt badly.

5. I tend to help others (i.e., family/friends/ strangers) particularly when they are emotionally distressed.

6. It is easy for me to help others (i.e., family/friends/strangers) when they are in a bad situation.

7. I respond to helping others (i.e., family/friends/strangers) best when the situation is highly emotional.

8. I never hesitate to help others (i.e., family/friends/strangers) when they ask for it.

9. Emotional situations make me want to help others (i.e., family/friends/strangers) in need. 


\section{VITA}

Sarah L. Pierotti was born in Warren, Pennsylvania and spent most of her childhood growing up in Western Pennsylvania. After graduating from Butler Senior High School in Butler, Pennsylvania in 2009, she earned a Bachelor of Science with Honors in Psychology and Minor in Sociology from the University of Pittsburgh in Pittsburgh, Pennsylvania in 2013, where she began to study prosocial behaviors in early childhood in Dr. Celia Brownell's Early Social Development Lab. For her post-graduate education, she attended the University of MissouriColumbia starting in 2013 in the Department of Human Development and Family Science. She earned a Master of Science in Early Childhood Development in 2016, completed her comprehensive exams for the doctoral degree in 2017, and is graduating with the Doctorate of Philosophy in Human Environmental Sciences in August 2020. 